\title{
Polynomial-clone Reducibility
}

\author{
Quinn Culver* \\ Department of Mathematics
}

15 June 2010

\begin{abstract}
Polynomial-clone reducibilities are generalizations of the truth-table reducibilities. A polynomial clone is a set of functions over a finite set $X$ that is closed under composition and contains all the constant and projection functions. For a fixed polynomial clone $\mathcal{C}$, a sequence $B \in X^{\omega}$ is $\mathcal{C}$-reducible to $A \in X^{\omega}$ if there is an algorithm that computes $B$ from $A$ using only effectively selected functions from $\mathcal{C}$. We show that if $A$ is a Kurtz random sequence and $\mathcal{C}_{1} \nsubseteq \mathcal{C}_{2}$ are distinct polynomial clones, then there is a sequence $B$ that is $\mathcal{C}_{1}$-reducible to $A$ but not $\mathcal{C}_{2}$-reducible to $A$. This implies a generalization of a result first proved by Lachlan for the case $|X|=2$. We also show that the same result holds if Kurtz random is replaced by Kolmogorov-Loveland stochastic.
\end{abstract}

\section{Contents}

1 Introduction $\quad 2$

2 Preliminaries 2

3 Polynomial Clone Reducibility $\quad 8$

4 Random Sequences $\quad 9$

5 KL-immunity and KL-stochasticity 19

6 Additional Results 25

7 Open Questions $\quad 26$

*Department of Mathematics, University of Hawai'i at Mānoa, 2565 McCarthy Mall, Honolulu, HI 96822, U.S.A., quinn@math.hawaii.edu. This material is based upon work supported by the National Science Foundation under Grant No. 0901020, and forms part of the author's Master's thesis at the University of Hawai'i at Mānoa. The Master's Committee consisted of Bjørn Kjos-Hanssen (chair) and Ralph Freese. 


\section{Acknowledgments}

My primary thanks go to my primary advisor, Dr. Bjørn Kjos-Hanssen for teaching me computability theory and algorithmic randomness and for always giving me something further to consider. Secondary thanks go to my secondary advisor, Dr. Ralph Freese for helping me to work out the details of some of my arguments and answering all my questions regarding lattice theory and universal algebra. Ternary thanks go to Dr. David Ross for getting me interested in mathematical logic in the first place. Remaining thanks go to the remainder of the UH Mānoa mathematics department.

\section{Introduction}

In this paper we address a generalization of truth-table reducibility and use notions of algorithmic randomness to prove facts about the it. We start by motivating our work with a bit of background, especially regarding many-one reducibility, truth-table reducibility, and the reducibilities in between. Then we use a concept from universal algebra, namely that of a polynomial clone, to define a generalization of those reducibilities, the key difference being that in this generalization there are potentially uncountably many reducibilities. We define Kurtz randomness in terms of betting strategies (martingales) and use it to show that there are indeed uncountably many new reducibilities and moreover, the sets on which they collapse are small in both a measure-theoretic and Bairecategorical sense.

Then we address the same problem via Kolmogorov-Loveland (KL) stochasticity, which is incomparable with Kurtz randomness. KL-stochasticity is weakened to a concept which we call KL-immunity and show that the results obtained for Kurtz randomness hold also for KL-immunity.

Using the so called truth-table cylinders, we show that there are times when all the continuum-many reducibilities collapse. The prior results show, however, that the set on which this happens is both measure-theoretically and Bairecategorically small.

Finally, we address some open questions regarding the work we have done. The first regards a common refinement between Kurtz randomness and KLimmunity. The second regards a strengthening of KL-stochasticity and an idea for its application to polynomial-clone reducibilities over a two-element set.

\section{Preliminaries}

\section{Notation}

The notation herein is mostly standard. We use $\omega$ to denote the set of natural numbers and $\omega^{+}$the set of positive natural numbers. The set of all infinite sequences over a set $X$ is denoted $X^{\omega}$. The set of all finite strings over $X$ is denoted $X^{<\omega}$. The symbol $\varnothing$ denotes the empty string. If $\sigma, \tau \in X^{<\omega}$, then $\sigma \tau$, 
or $\sigma^{\frown} \tau$, denotes their concatenation and $|\sigma|$ the length of $\sigma$; i.e. if $\sigma=s_{1} \ldots s_{n}$, then $|\sigma|=n$. The relationship $\tau \prec \sigma$ means that $\sigma=\tau \rho$ for some $\rho \neq \varnothing$ and $\tau \preceq \sigma$ means $\tau \prec \sigma$ or $\tau=\sigma$. For any word $\sigma=s_{1} s_{2} \cdots s_{n}$, the notation $\sigma \uparrow k$ indicates the word $s_{1} s_{2} \cdots s_{k}$ consisting of the first $k$ bits of $\sigma$ and is called a prefix or initial segment of $\sigma$; the string $\tau$ is a prefix of $\sigma$ iff $\sigma$ extends $\tau$. Similarly, $\sigma \uparrow_{i}^{j}=s_{i} \cdots s_{j}$.

For any $A \in X^{\omega}, A \uparrow k$ means the word $A(0) A(1) \cdots A(k-1)$ consisting of the first $k$ bits of $A$ and is called a prefix or initial segment of $A$. When $\sigma \in X^{<\omega}$, we will also write $\sigma \prec A$ when $A(0) \cdots A(|\sigma|-1)=\sigma$ or $\sigma=\varnothing$. It will be useful to consider the set of all sequences that extend a given string, so for any $\sigma \in X^{<\omega}$, we define $[\sigma]=\left\{A \in X^{\omega}: \sigma \prec A\right\}$.

We will use strings and tuples interchangeably as arguments of functions. For example, if $f$ is a $k$-ary function and $\sigma=s_{1} s_{2} \cdots s_{k}$, then $f(\sigma), f\left(s_{1}, s_{2}, \ldots, s_{k}\right)$, and $f\left(\sigma\left\lceil j, s_{j+1}, \ldots, s_{k}\right)\right.$ all mean the same thing.

\section{Cantor Space}

Let $\mathbf{n}=\{0,1, \ldots, n-1\}$. From now on, $n \geq 1$ is fixed. We will be working over the generalized Cantor space $\mathbf{n}^{\omega}$. We assume knowledge of point-set topology, including Baire category theory, and also knowledge of basic measure theory. Familiarity with the concepts covered in Royden's Real Analysis [14] is sufficient.

The space $\mathbf{n}^{\omega}$ is equipped with a topology whose basis consists of the clopen sets $[\sigma]$, for $\sigma \in \mathbf{n}^{<\omega}$. The space $\mathbf{n}^{\omega}$ is also equipped with a measure $\mu$, that is defined on the basis by setting $\mu([\sigma])=n^{-|\sigma|}$ for each $\sigma \in \mathbf{n}^{<\omega}$ (so in particular $\left.\mu\left(\mathbf{n}^{\omega}\right)=2^{0}=1\right)$.

A set $S$ of strings is called prefix-free if $\sigma, \tau \in S \Rightarrow \sigma \nprec \tau$. The following obvious but useful fact states that every open set can be generated by a prefixfree set.

Fact 1. For every open set $O \subset \mathbf{n}^{\omega}$, there exists a prefix-free set $S \subset \mathbf{n}^{<\omega}$ such that $O=\bigcup_{\sigma \in S}[\sigma]$.

\section{Computability Theory}

We assume a working knowledge of the basics of computability theory. Specifically, the concepts of computable functions, computable sets, computably enumerable (c.e.) sets, undecidability of the halting problem, Church's thesis, and Gödel (aka effective) numberings will be used or mentioned but not defined/proven. As is customary within computability theory, we will not rigorously prove that given objects are computable, but will instead provide an argument as to their effective calculabilty and appeal to Church's thesis. See Cutland's monograph [3] or Odifreddi's [10] for details on Church's thesis or any of the other aforementioned computability topics. 


\section{Reducibility}

The central theme of this paper is a special type of reducibility, so we will provide a brief introduction to reducibility for the reader unfamiliar with the topic and its role in computability theory. Reductions play a central part in computability theory because they characterize how some problems are "harder" than others. Specifically, if $A$ and $B$ are sets (of natural numbers), a reduction from $A$ to $B$ shows that the problem of determining membership in $A$ can be effectively reduced to determining membership (of usually different elements) in $B$. So it is in this sense that the problem (of determining membership in) $A$ is no harder than the problem $B$. In this context the set $B$ is called an oracle. For example, the word problem for groups was shown unsolvable by a reduction to it from the halting problem [13, chapter 12]. Thus the halting problem is no harder than the word problem for groups; if the word problem for groups were solvable, so then would be the halting problem.

The most general (and still practical) reducibility is Turing reducibility. Although Turing reducibility is of great interest in computability theory, it is not of interest here. We investigate reducibilities that are strictly stronger than Turing reducibility, the strongest of which is many-one reducibility.

Definition 2. A set $A \subseteq \omega$ is m-reducible to $B \subseteq \omega$, written $A \leq_{m} B$ if there is a computable function $f: \omega \rightarrow \omega$ such that $x \in A$ if and only if $f(x) \in B$.

If $A \leq_{m} B$, then $A$ is no harder than $B$ because determining membership in $A$ reduces to determining membership in $B$ (via $f$ ). The relation $\leq_{m}$ is reflexive (because the identity function is computable) and transitive (because the composition of computable functions is computable) and hence induces an equivalence relation $\equiv_{m}$, where $A \equiv_{m} B$ means $A \leq_{m} B$ and $B \leq_{m} A$. The corresponding equivalence classes are called $m$-degrees. The relation $\leq_{m}$ induces a partial ordering on the $m$-degrees that is in fact a distributive upper (i.e. join) semilattice of cardinality $2^{\aleph_{0}}$. From an $m$-reducibility standpoint, the sets $\emptyset$ and $\omega$ are uninteresting nuisances that require distracting provisos. In particular, $A \leq_{m} \omega$ (resp. $A \leq_{m} \emptyset$ ) if and only if $A=\omega$ (resp. $A=\emptyset$ ). Thus the $m$-degrees of $\emptyset$ and $\omega$ are disregarded and now the semilattice of $m$-degrees has a minimum element, the $m$-degree of all computable sets [10].

There are drawbacks to $m$-reducibility. Since reducibility is intended to capture the essence of " $A$ is no harder than $B$ ", it should definitely be the case that any set $A$ is reducible to its complement $A^{\complement}$ because the membership question "is $x \in A$ ?" can be reduced to "is $x \notin A^{\mathrm{C}}$ ?". However, if $A$ is any c.e. set that is not computable (such as the halting set $K$ ), then $A \Varangle_{m} A^{\complement}$ and

$A^{\complement} \leq_{m} A$. To see why, first notice that $A \leq_{m} A^{\complement}$ if and only if $A^{\complement} \leq_{m} A$ (via the same computable function). Then $A^{\complement} \leq_{m} A$ implies that $A^{\complement}$ is c.e. and hence $A$ is in fact computable (because $A$ is computable if and only if $A$ and $A^{\complement}$ are c.e.).

The problem with $m$-reducibility just described stems from the fact that it reduces $A$ 's membership question "is $x$ in $A$ ?" to a single, very specific query to the oracle $B$, "is $f(x)$ in $B$ ?" So we introduce a more general reducibility, 
truth-table reducibility, which allows $A$ 's membership question to be reduced to any finite Boolean combination of queries to $B$.

Before defining truth-table reducibility, we shift from working with sets to sequences. This is really no change since to every set $A \subseteq \omega$ corresponds the characteristic sequence $C_{A} \in 2^{\omega}$, where $C_{A}(i)=1 \Leftrightarrow i \in A$. A set $A$ and its characteristic sequence $C_{A}$ are identified, so we can dually think of and refer to $A$ as both a sequence and a set. Note that in this setting, $A \leq_{m} B$ if and only if there is a computable $f: \omega \rightarrow \omega$ such that $A(i)=B(f(i))$ for each $i$.

A Boolean operation is a function $f:\{0,1\}^{k} \rightarrow\{0,1\}$ for some $k$. Since for each $k$ there are only finitely many Boolean operations from $\{0,1\}^{k}$ into $\{0,1\}$, there are countably many Boolean operations. We order the set of all Boolean operations by first designating that functions with domain $\{0,1\}^{k_{1}}$ precede those with domain $\{0,1\}^{k_{2}}$ whenever $k_{1}<k_{2}$. For a given $k$, to order the functions with domain $\{0,1\}^{k}$, we first order the $2^{k}$ binary strings of length $k$ lexicographically so that to each function $f:\{0,1\}^{k} \rightarrow\{0,1\}$ corresponds the string of length $2^{k}$, whose $i^{\text {th }}$ element is the image under $f$ of the lexicographically $i^{\text {th }}$ length- $k$ string. We shall call this the image string of $f$. Then if $f_{1}, f_{2}:\{0,1\}^{k} \rightarrow\{0,1\}$, we designate that $f_{1}$ precedes $f_{2}$ the image string of $f_{1}$ lexicographically precedes the image string of $f_{2}$.

The ordering just described is effective: given $i$, the $i^{\text {th }}$ Boolean operation can be computed and, conversely, given a Boolean operation, its position in the ordering can be computed. This makes sense of the phrase "a computable sequence of Boolean operations". A sequence $\left\langle h_{i}\right\rangle_{i \in \omega}$ of Boolean operations is computable if and only if there is a computable function $f$ such that $f(i)=j$ whenever $h_{i}$ is the $j^{\text {th }}$ Boolean operation in the ordering above.

Definition 3. A set $A \subseteq \omega$ is tt-reducible to $B \subseteq \omega$, written $A \leq_{t t} B$, if there is a computable sequence $\left\langle h_{i}\right\rangle_{i \in \omega}$ of Boolean operations such that

$$
A(i)=h_{i}\left(B \nmid a_{i}\right),
$$

for each $i \in \omega$, where $a_{i}$ is the arity of $h_{i}$.

Just as with $m$-reducibility the relation $\leq_{t t}$ is reflexive (via projection functions) and transitive (because $\left\langle g_{i}\right\rangle_{i \in \omega}$ is closed under composition) and hence induces the equivalence relation $\equiv_{t t}$, where $A \equiv_{t t} B$ means $A \leq_{t t} B$ and $B \leq_{t t} A$. The corresponding equivalence classes are called $t$ t-degrees. The relation $\leq_{t t}$ induces a partial ordering on the $t t$-degrees that is an upper semilattice of cardinality $2^{\aleph_{0}}[10]$.

Truth-table reducibility is more general than $m$-reducibility. Indeed, Proposition 4 below describes the exact relationship between the two reducibilities.

Proposition 4. Let $A \subseteq \omega$ and $\emptyset \subsetneq B \subsetneq \omega$. Then $A \leq_{m} B$ if and only if there is a computable sequence $\left\langle h_{i}\right\rangle_{i \in \omega}$ consisting only of projection and constant functions such that $A(i)=h_{i}\left(B\left\lceil a_{i}\right)\right.$ for each $i \in \omega$, where $a_{i}$ is the arity of $h_{i} \cdot{ }^{1}$

${ }^{1}$ Interestingly, Proposition 4 suggests a "better" definition of $m$-reducibility than given in 
Proof. Suppose $A \leq_{m} B$ with $i \in A \Leftrightarrow f(i) \in B$, for some computable $f$. Then $A(i)=\pi_{f(i)+1}^{f(i)+1}(B \nmid f(i)+1)$ for each $i \in \omega$, where $\pi_{u}^{v}$ denotes the $v$ ary projection onto the $u^{\text {th }}$ variable. The sequence $\left\langle\pi_{f(i)+1}^{f(i)+1}\right\rangle_{i \in \omega}$ is computable because $f$ is.

Now suppose there is a computable sequence $\left\langle h_{i}\right\rangle_{i \in \omega}$ consisting only of projection and constant functions such that $A(i)=h_{i}\left(B \uparrow a_{i}\right)$, for each $i \in \omega$. Fix $b \in B$ and $c \in B^{\complement}$. Whenever $h_{i}$ is the projection $\pi_{u}^{v}$, define $f(i)=h_{i}(B \nmid v)$. Whenever $h_{i}$ is the constant 1 , define $f(i)=b$. Whenever $h_{i}$ is the constant 0 , define $f(i)=c$. Then clearly $f$ is computable and $i \in A$ if and only if $A(i)=1$ if and only if $B(f(i))=1$ if and only if $f(i) \in B$.

With the characterization of $m$-reducibility given in Proposition 4 in mind, $m$-reducibility is conspicuously similar to $t t$-reducibility. The only difference is the universe of Boolean operations whence functions in the computable sequence can come; $t t$-reducibility's universe consists of all Boolean operations whereas $m$-reducibility's universe consists of only the constants and projections. It is, therefore, natural to wonder which other sets of Boolean operations could serve as an appropriate universe for reducibility.

Recalling that a reduction from $A$ to $B$ means that $A$ is no harder than $B$, it is manifestly necessary that any reducibility $\leq_{r}$ relation have the following three properties:

i. reflexivity,

ii. transitivity,

iii. $A$ computable $\Rightarrow A \leq_{r} B$ for any set $B$.

Both $m$ - and $t t$-reducibilities have all three properties. The reasons are, respectively, because both their universes

1. contain all projection functions,

2. are compositionally closed, and

3. contain all constant functions.

Sets of functions that satisfy properties 1-3 are called polynomial clones and are of special interest in universal algebra. Of course, the underlying set need not be $\{0,1\}$.

Definition 2, which is the one given in texts (cf. [3] or [10]). If $m$-reducibility is defined by $A \leq_{m} B$ iff there is a computable sequence $\left\langle h_{i}\right\rangle_{i \in \omega}$ consisting only of projection and constant functions such that $A(i)=h_{i}\left(B\left\lceil a_{i}\right)\right.$ for each $i \in \omega$, where $a_{i}$ is the arity of $h_{i}$, then the sets $\emptyset$ and $\omega$ no longer have the singular properties $A \leq_{m} \emptyset$ (resp. $A \leq_{m} \omega$ ) iff $A=\emptyset$ (resp. $A=\omega)$. In fact now it is the case that $A \leq_{m} \emptyset, \omega$ iff $A$ is computable, so the semilattice of $m$-degrees has a minimum element without removing the degrees of $\emptyset$ and $\omega$. 
Definition 5. An operation on a set $X$ is a function $f: X^{n} \rightarrow X$ for some $n$. A clone (closed operation network) over $X$ is a set of operations on $X$ that is closed under composition and contains all projections. A polynomial clone is a clone that also contains the constant functions.

In light of Definition 5, the reason the universes of $m$ - and $t t$-reducibilities are suitable for reducibility notions is that they are both polynomial clones over $\{0,1\}$. Post showed in [12] that there are exactly seven polynomial clones over $\{0,1\}$. Given any set $\mathcal{F}$ of Boolean operations, there is a smallest polynomial clone containing $\mathcal{F}$ called the polynomial clone generated by $\mathcal{F}$. The five Boolean polynomial clones other than the two already listed (the minimum and maximum ones) are generated by the sets $\{\vee\},\{\wedge\},\{\neg\},\{+\}$, and $\{\vee, \wedge\}$, where + is addition modulo 2 ; their corresponding reducibilities are $d$ (disjunctive), $c$ (conjunctive), btt(1) (bounded truth-table with norm 1), $\ell$ (linear), and $p$ (positive).

It is important to realize that all we have thus far deduced is that there are at most seven reducibilities satisfying properties i-iii between (and including) $m$ - and $t t$-reducibility. Lachlan [7] (also referenced by Bulitko in [2]) showed that there are in fact exactly seven reducibilities between (and including) $\mathrm{m}$ and $t t$-reducibility so no implications hold other than those indicated in Figure 1 (see also [11]). (In [11], Odifreddi also states that the same result follows from results of Jockusch [6] and Degtev [4].)

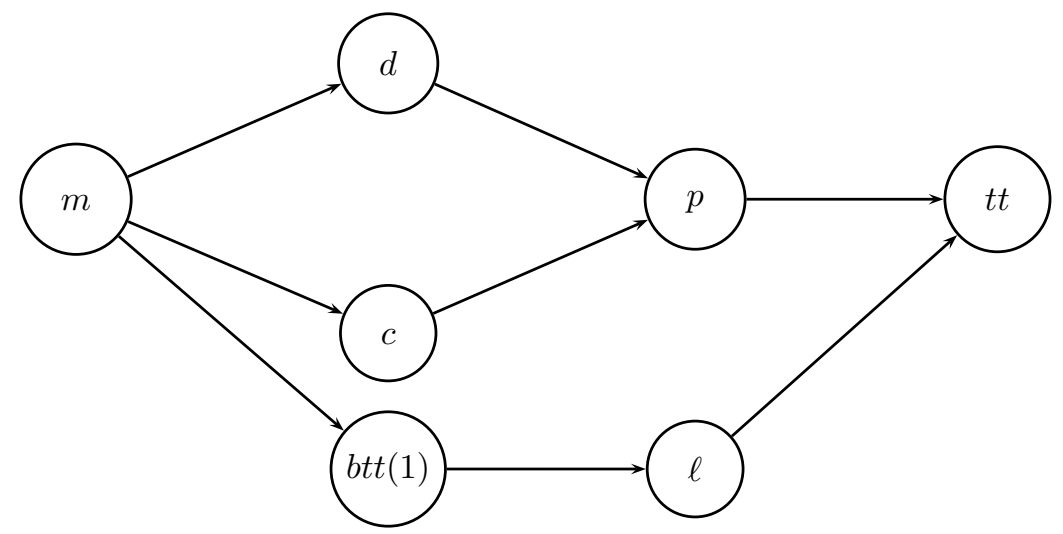

Figure 1: The implication lattice of reducibilities over $\{0,1\}$.

Instead of polynomial clones and sequences over $\{0,1\}$, we work here with clones over the general finite set $\mathbf{n}=\{0,1, \ldots, n-1\}$. Elements of $\mathbf{n}^{\omega}$ can be viewed as bounded functions of natural numbers.

Although the foregoing exhibits a simple structure of the lattice polynomial clones over $\{0,1\}$, there are uncountably many polynomial clones over $\mathbf{n}$ when $n \geq 3$ [1]. So the structure of the polynomial clones over $\mathbf{n}$ when $n \geq 3$, although still a lattice with minimum and maximum element, is very complex and still largely a mystery. 


\section{Polynomial Clone Reducibility}

Since the reducibilities $m, d, c, b t t(1), p, \ell$, and $t t$ can be expressed in terms of polynomial clones, we define similar reducibilities for sequences over $\mathbf{n}$. The set of all operations over $\mathbf{n}$ can be effectively numbered exactly as the Boolean operations. We assume a fixed effective numbering of all operations over $\mathbf{n}$ so we can speak of computable sequences of operations on $\mathbf{n}$.

Definition 6. Let $\mathcal{C}$ be a polynomial clone on $\mathbf{n}$ and $A, B \in \mathbf{n}^{\omega}$. Then $A$ is said to be $\mathcal{C}$-reducible to $B$, written $A \leq_{\mathcal{C}} B$, if there is a computable sequence $\left\langle h_{i}\right\rangle_{i \in \omega}$ from $\mathcal{C}$ such that for all $i$

$$
A(i)=h_{i}\left(B \nmid a_{i}\right),
$$

where $a_{i}$ is the arity of $h_{i}$.

In this scenario we say that $A \leq_{\mathcal{C}} B$ is witnessed by $\left\langle h_{i}\right\rangle_{i \in \omega} \subseteq \mathcal{C}$.

At first glance, Definition 6 might seem restrictive due to the requirement that the input of $h_{i}$ be the unique $a_{i}$-bit prefix of $B$. However, since we can always compose with projection functions, any of the inputs $B(0), \ldots, B\left(a_{i}-1\right)$ could be dummies. The definition given is just a clean way of expressing the fact that the value $A(i)$ can be computed by some effectively determinable operation on $\mathbf{n}$ applied to some effectively determinable bits of $B$.

Herein we exploit one particular instance of polynomial clone reducibility: for a given $f \in \mathcal{C}$ and $A \in \mathbf{n}^{\omega}$, let $f\{A\}$ be the sequence whose $i^{\text {th }}$ element is given by

$$
f\{A\}(i)=f(A(k i+1), \ldots, A(k i+k)),
$$

where $k$ is the arity of $f$. As one might expect, $f\{A\} \leq_{\mathcal{C}} A$, a fact that follows immediately from Definition 6 using the sequence $\left\langle h_{i}\right\rangle_{i \in \omega}$ of functions whose $i^{\text {th }}$ element is $f$ composed with projections:

$$
h_{i}\left(x_{0}, \ldots, x_{k i+k}\right)=f\left(\pi_{k i+2}^{k i+k+1}\left(x_{0}, \ldots, x_{k i+k}\right), \ldots, \pi_{k i+k+1}^{k i+k+1}\left(x_{0}, \ldots, x_{k i+k}\right)\right) .
$$

Notation. Whenever the symbol $f$ is used for a function in a polynomial clone $\mathcal{C}$, the notation $f\{A\}$ is shorthand for the sequence whose $i^{\text {th }}$ element is given by equation (1). If not stated explicitly otherwise, the arity of $f$ is $k$.

It is shown here that for distinct polynomial clones $\mathcal{C}_{1}$ and $\mathcal{C}_{2}$ with (a suitably chosen) $f \in \mathcal{C}_{1}-\mathcal{C}_{2}$, we can always find $A \in \mathbf{n}^{\omega}$ such that $f\{A\} \leq_{\mathcal{C}_{1}} A$ but $f\{A\} \not \mathcal{C}_{2} A$. Moreover, for any $\mathcal{C}_{1} \nsubseteq \mathcal{C}_{2}$ the set

$$
\mathrm{Eq}\left(\mathcal{C}_{1}, \mathcal{C}_{2}\right):=\left\{A \in \mathbf{n}^{\omega}: \forall B \in \mathbf{n}^{\omega}\left(B \leq_{\mathcal{C}_{1}} A \Rightarrow B \leq_{\mathcal{C}_{2}} A\right)\right\}
$$

is shown to be small in both the measure-theoretic (null) and Baire-categorical (meager) senses.

The next result, Lemma 7, allows us to work with a function that depends on its last variable $x_{k}$ and therefore on any segment $x_{j}, \ldots, x_{k}, j \leq k$; it rids us of pesky dummy variables. 
Lemma 7. If $\mathcal{C}_{1} \nsubseteq \mathcal{C}_{2}$, then there is a function $f \in \mathcal{C}_{1}-\mathcal{C}_{2}$ such that either $k=1$ or has $f$ the property that for any $j \in\{1, \ldots, k-1\}$ there are $x_{1}, \ldots, x_{k} \in \mathbf{n}$ and $y_{j+1}, \ldots, y_{k} \in \mathbf{n}$ such that

$$
f\left(x_{1}, \ldots, x_{k}\right) \neq f\left(x_{1}, \ldots, x_{j}, y_{j+1}, \ldots, y_{k}\right),
$$

where $k$ is the arity of $f$.

Proof. Let $f_{1} \in \mathcal{C}_{1}-\mathcal{C}_{2}$. If $f_{1}$ is essentially unary, then it can be composed with constants to obtain a unary function $f_{2} \in \mathcal{C}_{1}-\mathcal{C}_{2}$.

Suppose now that $f_{1}$ is not essentially unary and there is $j \in\{1, \ldots, k-1\}$ such that for any $x_{1}, \ldots, x_{k} \in \mathbf{n}$ and $y_{j+1}, \ldots, y_{k} \in \mathbf{n}$ we have

$$
f_{1}\left(x_{1}, \ldots, x_{k}\right)=f_{1}\left(x_{1}, \ldots, x_{j}, y_{j+1}, \ldots, y_{k}\right) .
$$

We assume $j$ has been minimally chosen. By defining

$$
f_{2}\left(x_{1}, \ldots, x_{j}\right)=f_{1}\left(x_{1}, \ldots, x_{j}, 0,0, \ldots, 0\right),
$$

we get a new function in $\mathcal{C}_{1}$. Moreover, $f_{2} \notin \mathcal{C}_{2}$ because $f_{1}=f_{2}\left(\pi_{1}^{k}, \ldots, \pi_{j}^{k}\right)$ and $j>1$ because $f_{1}$ is not essentially unary.

We claim that $f_{2}$ has the desired property. If there is $j^{\prime} \in\{1, \ldots, j-1\}$ such that for any $x_{1}, \ldots, x_{j} \in \mathbf{n}$ and any $y_{j^{\prime}+1}, \ldots, y_{j} \in \mathbf{n}$ the equality

$$
f_{2}\left(x_{1}, \ldots, x_{j}\right)=f_{2}\left(x_{1}, \ldots, x_{j^{\prime}}, y_{j^{\prime}+1}, \ldots, y_{j}\right) .
$$

holds, then for any $x_{1}, \ldots, x_{k} \in \mathbf{n}$ and $y_{j^{\prime}+1}, \ldots, y_{k} \in \mathbf{n}$ we have

$$
\begin{aligned}
f_{1}\left(x_{1}, \ldots, x_{k}\right) & =f_{2}\left(x_{1}, \ldots, x_{j}\right) \\
& =f_{2}\left(x_{1}, \ldots, x_{j^{\prime}}, y_{j^{\prime}+1}, \ldots, y_{j}\right) \\
& =f_{1}\left(x_{1}, \ldots, x_{j^{\prime}}, y_{j^{\prime}+1}, \ldots, y_{k}\right),
\end{aligned}
$$

contrary to $j$ 's minimality.

\section{Random Sequences}

Now we prove that the set $\operatorname{Eq}\left(\mathcal{C}_{1}, \mathcal{C}_{2}\right)$ (see equation (2) for the definition of $\left.\mathrm{Eq}\left(\mathcal{C}_{1}, \mathcal{C}_{2}\right)\right)$ has measure zero. This gives the intuitive result, distinct polynomial clones yield distinct reducibilities, a result already known for polynomial clones over $\{0,1\}$. To do so, we show that no random sequence is in $\operatorname{Eq}\left(\mathcal{C}_{1}, \mathcal{C}_{2}\right)$. The result follows because the collection nonrandom sequences has measure zero. So in addition to generalizing the abovementioned result, our work also provides a nice introduction to the rudiments of algorithmic randomness and its applications.

Algorithmic randomness is the branch of computability theory that attempts to define what it means for an infinite sequence to be random. People commonly identify randomness with the property of unpredictability. There are, however, 
other properties that make their own case for characterizing randomness. For example, a random sequences should be "incompressible" (via Kolmogorov complexity) and "common" amongst other sequences (in a measure-theoretic sense). Fortunately, the most popular definition of randomness, Martin-Löf (ML) randomness, can be characterized using unpredictability, incompressibility, or commonality. We will work with a weaker randomness than that of Martin-Löf, called Kurtz randomness. This is no loss since we will be showing that sequences with certain properties are not Kurtz random, so all our results hold for ML random sequences (and those in between) as well.

Kurtz randomness is typically defined in terms of the commonality property, but can also be defined in terms of the unpredictability property. We will use unpredictability, which is formalized in Definition 8. For more details on algorithmic randomness, see [5] or [9].

As a warm-up for Definition 8, consider the infinite game whose goal is to make money by betting on successive bits of $A \in \mathbf{n}^{\omega}$. The bits of $A$ will be revealed in order, beginning with $A(0)$, and we can use our knowledge of $A\lceil i$ to bet on $A(i)$. We can divide our current capital as we please among the $n$ possibilities for $A(i)$ and the house will return $n$ times a correct bet. For example, suppose we have seen $A \uparrow 5$, our current capital is 1 and (somehow) we know that $A(5)$ is 0 or $n-1$. We bet $1 / 2$ that $A(5)=0$ and $1 / 2$ that $A(5)=n-1$, guaranteeing a payback of $n / 2$. If $n>2$, we have made money. (Otherwise, our information was a mere truism.) Informally, a sequence $A \in \mathbf{n}^{\omega}$ is random if there is no effective betting strategy that guarantees limitless winnings in the game.

Betting strategies are represented by their capital functions, called martingales. For example, in the above scenario our martingale would look like $M\left(\sigma^{\urcorner} 0\right)=\frac{n}{2} M(\sigma)=M\left(\sigma^{\wedge} n\right)$ and $M\left(\sigma^{\wedge} i\right)=0$ for each $\sigma$ with $|\sigma|=5$ and each $i \in \mathbf{n}-\{0, n-1\}$. Note that $n M(\sigma)=\sum_{i \in \mathbf{n}} M\left(\sigma^{\frown} i\right)$.

Definition 8. ([16], see also [5])

(i) A (computable) martingale is a (computable) function $M: \mathbf{n}^{<\omega} \rightarrow \mathbb{R}^{+} \cup\{0\}$ such that

$$
M(\sigma)=\frac{M\left(\sigma^{\frown} 0\right)+M\left(\sigma^{\frown} 1\right)+\cdots+M\left(\sigma^{\frown}(n-1)\right)}{n}
$$

for each $\sigma \in \mathbf{n}^{<\omega}$.

(ii) A (computable) martingale $M$ succeeds on $A \in \mathbf{n}^{\omega}$ if

$$
\limsup _{i \rightarrow \infty} M(A\lceil i)=\infty .
$$

We write $S(M)$ for the set of all elements of $\mathbf{n}^{\omega}$ on which the martingale $M$ succeeds.

(iii) A sequence $A \in \mathbf{n}^{\omega}$ is Kurtz random if there is no computable martingale $M$ that succeeds on $A$ with $M(A\lceil i) \geq d(i)$ for all $i$ for some 
nondecreasing unbounded computable function $d: \omega \rightarrow \omega$. Equivalently stated, a sequence $A \in \mathbf{n}^{\omega}$ is not Kurtz random if and only if there is a computable martingale $M$ and a nondecreasing unbounded computable function $d: \omega \rightarrow \omega$ such that $M(A\lceil i) \geq d(i)$ for all $i$.

Kurtz randomness is a very weak form of randomness. Schnorr [15] (see also [5]) argued that for a sequence to be nonrandom, there should be some betting strategy that guarantees limitless winnings and such that if we follow the strategy we know precisely when we will reach a given capital. In this sense Kurtz nonrandomness is too restrictive, because not only must we know when we will reach a given capital, but our capital cannot dip arbitrarily low between peaks. However, if we were betting, and we knew that we would reach a capital of $M$ after betting on at most 25 more bits, we would not care how low our capital gets in the meantime, so Kurtz nonrandomness is intuitively too restrictive. We would like a nonrandom sequence to be succeeded upon by a capital function that looks, at worst, like $1+x \sin x$, but Kurtz nonrandomness requires a capital function that looks, at worst, like $x+x \sin x$.

Equation (3) is known as the fairness condition because it reflects the fact that the house will take our entire bet if we lose or return it multiplied by $n$ if we win. In order to proceed with to our main results, we need some facts about martingales, the first of which is the equation

$$
M(\sigma)=n^{-N} \sum_{|\tau|=N} M(\sigma \tau),
$$

which holds for all $\sigma \in \mathbf{n}^{<\omega}$ and follows immediately from the fairness condition. The next lemma is useful in proving Lemma 10, which shows that the success set of any martingale is null and is necessary for proving our main result, Theorem 18.

Lemma 9. If $S \subseteq \mathbf{n}^{<\omega}$ is prefix-free and $M$ is a martingale, then

$$
M(\varnothing) \geq \sum_{\sigma \in S} M(\sigma) n^{-|\sigma|}
$$

Proof. It suffices to show that for each $\tau \in S$,

$$
M(\varnothing) \geq \sum_{\substack{\sigma \in S \\|\sigma| \leq|\tau|}} M(\sigma) n^{-|\sigma|} .
$$

We proceed by induction on $|\tau|$. For $\tau \in S$ of minimum length, inequality (5) follows from equation (4) because

$$
M(\varnothing)=\sum_{\substack{\sigma \in \leq S \\|\sigma| \leq|\tau|}} M(\sigma) n^{-|\sigma|}+\sum_{\substack{\sigma \notin S \\|\sigma| \leq|\tau|}} M(\sigma) n^{-|\sigma|} .
$$


Suppose now that for some $\tau \in S$, the inequality

$$
M(\varnothing) \geq \sum_{\substack{\sigma \in S \\|\sigma| \leq|\tau|}} M(\sigma) n^{-|\sigma|}+\sum_{\substack{\sigma \nsucceq v \in S \\|\sigma|=|\tau|}} M(\sigma) n^{-|\sigma|}
$$

holds. (The notation $\sigma \nsucceq v \in S$ means that $\sigma$ does not extend any elements of $S$.) Let $N=\min \{|\sigma|: \sigma \in S \&|\sigma|>|\tau|\}-|\tau|$, so $N$ represents how many bits longer than $\tau$ is the next-longest string of $S$. For each $\sigma$ with $|\sigma|=\tau$, equation (4) gives

$$
M(\sigma)=\sum_{|v|=N} n^{-N} M(\sigma v)
$$

so that

$$
\begin{aligned}
\sum_{\substack{\sigma \nsucceq v \in S \\
|\sigma|=|\tau|}} M(\sigma) n^{-|\sigma|} & =\sum_{\substack{\sigma \nsucceq v \in S \\
|\sigma|=|\tau|}} \sum_{|v|=N} n^{-(N+|\sigma|)} M(\sigma v) \\
& =\sum_{\substack{\sigma \in S \\
|\sigma|=|\tau|+N}} M(\sigma) n^{-|\sigma|}+\sum_{\substack{\sigma \nsucceq v \in S \\
|\sigma|=|\tau|+N}} M(\sigma) n^{-|\sigma|},
\end{aligned}
$$

where the last equality holds because $S$ is prefix-free. Combining this with inequality (6) gives

$$
M(\varnothing) \geq \sum_{\substack{\sigma \in S \\|\sigma| \leq|\tau|+N}} M(\sigma) n^{-|\sigma|}+\sum_{\substack{\sigma \nsucceq v \in S \\|\sigma|=|\tau|+N}} M(\sigma) n^{-|\sigma|},
$$

and completes the induction.

Lemma 10. The set of sequences on which a martingale succeeds has measure zero; that is $\mu(S(M))=0$ for every martingale $M$.

Proof. Fix $M$ and set $S_{m}=\left\{X \in \mathbf{n}^{\omega}: \exists i(M(X\lceil i)>m)\}\right.$. We show, following [16], that $\mu\left(S_{m}\right) \leq \frac{M(\varnothing)}{m}$. This is sufficient since $S(M)=\bigcap_{m} S_{m}$, so $\mu[S(M)] \leq$ $\mu\left(S_{m}\right)$ for each $m$.

First we show that $S_{m}$ is open (hence measurable). The equation

$$
\left\{X \in \mathbf{n}^{\omega}: M(X\lceil i) \geq m\}=\bigcup_{\substack{|\sigma|=i \\ M(\sigma) \geq m}}[\sigma]\right.
$$

shows that for any fixed $i$ and $m$, the set $\left\{X \in \mathbf{n}^{\omega}: M(X \uparrow i) \geq m\right\}$ is actually clopen because it is a finite union of clopen sets. Because $S_{m}=\bigcup_{i \in \omega}\{X \in$ $\mathbf{n}^{\omega}: M(X\lceil i) \geq m\}$, it follows that $S_{m}$ is open (and hence measurable). 
Since $S_{m}$ is open, Fact 1 implies that we can write $S_{m}=\bigcup_{i \in \omega}\left[\sigma_{i}\right]$, where the set $\left\{\sigma_{i}: i \in \omega\right\}$ is prefix-free and $M\left(\sigma_{i}\right) \geq m$ for each $i$. Note that $\mu\left(S_{m}\right)=$ $\sum_{i=0}^{\infty} n^{-\left|\sigma_{i}\right|}$. Then

$$
\begin{aligned}
M(\varnothing) & \geq \sum_{i \in \omega} M\left(\sigma_{i}\right) n^{-\left|\sigma_{i}\right|} \quad(\text { by Lemma 9) } \\
& \geq \sum_{i \in \omega} m n^{-\left|\sigma_{i}\right|} \\
& =m \sum_{i \in \omega} n^{-\left|\sigma_{i}\right|} \\
& =m \cdot \mu\left(S_{m}\right)
\end{aligned}
$$

so $\mu\left(S_{m}\right) \leq \frac{M(\varnothing)}{m}$ as desired.

Corollary 11. The collection of all Kurtz random sequences has measure one.

Proof. Since there are only countably many computable martingales and each one succeeds only on a set of measure zero, we have

$$
\mu\left[\bigcup_{M} S(M)\right] \leq \sum_{M} \mu[S(M)]=0 .
$$

Thus the collection of all Kurtz random sequences, which contains the measureone set $\mathbf{n}^{\omega}-\bigcup_{M} S(M)$, has measure one.

The next fact will be key in proving Lemmas 13-17, which together prove our main result, Theorem 18.

Lemma 12. Let $A \in \mathbf{n}^{\omega}$ and $k \in \omega$, let $n_{0}<n_{1}<\cdots$ be a computable sequence, and let $\left\langle m_{i}\right\rangle_{i \in \omega}$ be a computable sequence such that $k \leq m_{i} \leq k\left(n_{i+1}-n_{i}\right)$ for each $i \in \omega$. If there is a computable function $\Phi: \mathbf{n}^{\omega} \rightarrow \mathbf{n}^{\omega}$ such that

- if $|\sigma|=k n_{i}+1$, then $|\Phi(\sigma)|=n^{m_{i}-k} m_{i}$,

- and if $\sigma=A\left\lceil k n_{i}+1\right.$ then $\Phi(\sigma) \uparrow_{(j-1) m_{i}+1}^{j m_{i}} \neq A\left(k n_{i}+1\right) \cdots A\left(k n_{i}+m_{i}\right)$ for $j \in\left\{1, \ldots, n^{m_{i}-k}\right\}$,

then $A$ is not Kurtz random.

Betting strategy. We think of the bits of $A$ as representing the results of successive flips of an $n$-sided coin and describe a betting strategy that guarantees limitless winnings.

Having seen $A(0) \cdots A\left(k n_{i}\right)$ we compute $\Phi\left(A(0) \cdots A\left(k n_{i}\right)\right)$ and then can, by hypothesis, effectively rule out $n^{m_{i}-k}$ possibilities for $A\left(k n_{i}+1\right) \cdots A\left(k n_{i}+\right.$ $\left.m_{i}\right)$. We distribute our capital evenly amongst the remaining $n^{m_{i}}-n^{m_{i}-k}=$ $n^{m_{i}}\left(\frac{n^{k}-1}{n^{k}}\right)$ possibilities; so letting our capital be 1 , we are betting $\frac{1}{n^{m_{i}}\left(\frac{n^{k}-1}{n^{k}}\right)}$ on each possibility. Since exactly one of them will be correct and our odds 
(according to the house) are $\frac{1}{n^{m_{i}}}$, we are guaranteed a return of $n^{m_{i}}$ times our bet; i.e. we will win $n^{m_{i}} \frac{1}{n^{m_{i}}\left(\frac{n^{k}-1}{n^{k}}\right)}=\frac{n^{k}}{n^{k}-1}>1$. Since we can follow this strategy for each $i \in \omega$ and $\left(\frac{n^{k}}{n^{k}-1}\right)^{i} \rightarrow \infty$ as $i \rightarrow \infty$, we are guaranteed to win limitlessly.

Remark. Although the betting strategy just described does indeed guarantee unbounded capital, it does not guarantee that our capital will dominate an unbounded, increasing computable function as the definition of Kurtz nonrandomness requires. To remedy this we employ the so-called savings trick: instead of distributing all of our capital amongst the remaining possibilities, we distribute only half our current capital. The savings trick is reflected in the martingale construction below.

Martingale Construction. We recursively construct a computable martingale $M$ such that

$$
M\left(A\left\lceil k n_{i}+m_{i}+1\right)=\left(\frac{1}{2}\left[1+\frac{n^{k}}{n^{k}-1}\right]\right)^{i+1},\right.
$$

$M\left(A\lceil j) \geq \frac{1}{2}\right.$ for $j \leq k n_{0}+1$, and $M\left(A\lceil j) \geq \frac{1}{2}\left(\frac{1}{2}\left[1+\frac{n^{k}}{n^{k}-1}\right]\right)^{i}\right.$ for $k n_{i}+1<$ $j \leq k n_{i+1}+1$. This is sufficient because (a) $\left(\frac{1}{2}\left[1+\frac{n^{k}}{n^{k}-1}\right]\right)^{i+1} \rightarrow \infty$ as $i \rightarrow \infty$, so $M$ succeeds on $A$ and (b) the function $d: \omega \rightarrow \omega$ defined by $d(j)=\frac{1}{2}$ when $j \leq k n_{0}+1$ and $d(j)=\frac{1}{2}\left(\frac{1}{2}\left[1+\frac{n^{k}}{n^{k}-1}\right]\right)^{i}$ whenever $k n_{i}+1<j \leq k n_{i+1}+1$ is increasing, unbounded, computable, and has the property that $M(A\lceil j) \geq d(j)$ for all $j$.

Let $M(\sigma)=1$ for $|\sigma| \leq k n_{0}+1$. This reflects that our initial capital is 1 and we do not bet until at least having seen $A(0) \cdots A\left(k n_{0}\right)$.

Let $\alpha=n^{m_{0}-k}$ and fix $\sigma$ with $|\sigma|=k n_{0}+1$. For $j \in\{1, \ldots, \alpha\}$ let $\tau_{j}=\Phi(\sigma) \Upsilon_{(j-1) m_{0}+1}^{(j) m_{0}}$ so that (by hypothesis) if $A(0) \cdots A\left(k n_{0}\right)=\sigma$, then $A\left(k n_{0}+1\right) \cdots A\left(k n_{0}+m_{0}\right) \neq \tau_{j}$ for each $j$. Set $T=\left\{\tau_{j}: 1 \leq j \leq \alpha\right\}$.

For each $\rho$ with $0 \leq|\rho| \leq m_{0}$, let

$$
r_{\rho}=\mid\left\{v \in \mathbf{n}^{m_{0}}: \rho \preceq v \text { and } v \notin T\right\} \mid ;
$$

in words, $r_{\rho}$ is the number of strings that extend $\rho$ and are possibilities for $A\left(k n_{0}+1\right) \cdots A\left(k n_{0}+1\right)$. The two properties

$$
\begin{aligned}
& r_{\rho}=\sum_{s \in \mathbf{n}} r_{\rho s}, \\
& r_{\rho} \geq 1 \text { if } \rho \prec A\left(k n_{0}+1\right) \cdots A\left(k n_{0}+m_{0}\right)
\end{aligned}
$$

follow immediately from the definition of $r_{\rho}$.

Now for each $\rho$ with $0 \leq|\rho| \leq m_{0}$, define

$$
M(\sigma \rho)=\frac{1}{2}+\frac{1}{2} n^{|\rho|} \frac{r_{\rho}}{n^{m_{0}}-\alpha} .
$$


Note that if $|\rho|=0$, then $r_{\rho}=n^{m_{0}}-\alpha$ and so $M(\sigma)=M(\sigma \rho)=\frac{1}{2}+$ $\frac{1}{2} n^{0} \frac{n^{m_{0}-\alpha}}{n^{m_{0}-\alpha}}=1$, which is consistent with the previous definition of $M(\sigma)$. Define the function $C_{T}: \mathbf{n}^{<\omega} \rightarrow\{0,1\}$ to be 0 on $T$ and 1 on $T^{\complement}$. Then when $|\rho|=m_{0}$ we have

$$
\begin{aligned}
M(\sigma \rho)=\frac{1}{2}+\frac{1}{2} n^{m_{0}} \frac{C_{T}(\rho)}{n^{m_{0}}-\alpha} & =\frac{1}{2}+\frac{1}{2} n^{m_{0}} \frac{C_{T}(\rho)}{n^{m_{0}}-n^{m_{0}-k}} \\
& = \begin{cases}0 & \text { if } \rho \in T \\
\frac{1}{2}+\frac{1}{2} \cdot \frac{n^{k}}{n^{k}-1} & \text { if } \rho \notin T .\end{cases}
\end{aligned}
$$

Since $A\left(k n_{0}+1\right) \cdots A\left(k n_{0}+m_{0}\right) \notin T$, we have (so far) kept our promise that

$$
M\left(A\left\lceil k n_{0}+m_{0}+1\right)=\left(\frac{1}{2}\left[1+\frac{n^{k}}{n^{k}-1}\right]\right)^{0+1} .\right.
$$

For each $\sigma$ with $k n_{0}+m_{0}+1<|\sigma| \leq k n_{1}+1$, define $M(\sigma)=M(\sigma \mid$ $\left.k n_{0}+m_{0}+1\right)$. Our second promise, that $M\left(A\lceil j) \geq \frac{1}{2}\right.$ for $j \leq k n_{1}+1$, has now been fulfilled.

The computability of $M$-as constructed so far-follows from the fact that its components, $r_{\rho}, m_{0}$, and $\alpha$ are computable.

Now we verify that $M$-as constructed so far-satisfies the fairness condition, equation (3). For any $\rho$ with $|\rho|<m_{0}$ we have

$$
\begin{aligned}
\frac{1}{n} \sum_{s \in \mathbf{n}} M(\sigma \rho s) & =\frac{1}{n} \sum_{s \in \mathbf{n}}\left[\frac{1}{2}+\frac{1}{2} n^{|\rho s|} \frac{r_{\rho s}}{n^{m_{0}}-\alpha}\right] \\
& =\frac{1}{2}+\frac{1}{2} \cdot \frac{1}{n} \sum_{s \in \mathbf{n}} n^{|\rho|+1} \frac{r_{\rho s}}{n^{m_{0}}-\alpha} \\
& =\frac{1}{2}+\frac{1}{2} \cdot \frac{1}{n} \cdot \frac{n^{|\rho|+1}}{n^{m_{0}}-\alpha} \sum_{s \in \mathbf{n}} r_{\rho s} \\
& =\frac{1}{2}+\frac{1}{2} n^{|\rho|} \frac{r_{\rho}}{n^{m_{0}}-\alpha} \quad \text { (by equation (7)) } \\
& =M(\sigma \rho),
\end{aligned}
$$

so the fairness condition holds.

Note that the hypothesis $m_{0} \leq k\left(n_{1}-n_{0}\right)$ implies $k n_{0}+m_{0}+1 \leq k n_{1}+1$ and so $M$ has not yet been defined for those $\sigma$ such that $|\sigma|>k n_{1}+1$.

Suppose now $M$ has been defined for all words $\sigma$ with $|\sigma| \leq k n_{i+1}+1$ and that $M\left(A \uparrow k n_{i+1}+1\right)=\left(\frac{1}{2}\left[1+\frac{n^{k}}{n^{k}-1}\right]\right)^{i+1}$.

For each $\sigma$ with $|\sigma|=k n_{i+1}+1$, we can proceed mutatis mutandis as in the $i=0$ step, the primary difference being that for each $\rho$ with $0 \leq|\rho| \leq m_{i}$, we define

$$
M(\sigma \rho)=\frac{1}{2} M(\sigma)+\frac{1}{2} M(\sigma) n^{|\rho|} \frac{r_{\rho}}{n^{m_{i+1}}-\alpha} .
$$


Reasoning identical to the $i=0$ step shows that $M$ is computable, satisfies the fairness condition, $M\left(A \uparrow k n_{i+1}+m_{i+1}+1\right)=\left(\frac{n^{k}}{n^{k}-1}\right)^{i+2}$, and $M(A \uparrow$ $j) \geq \frac{1}{2}\left(\frac{1}{2}\left[1+\frac{n^{k}}{n^{k}-1}\right]\right)^{i+1}$ for $k n_{i+1}+1<j \leq k n_{i+2}+1$. This completes the construction.

Lemmas 13-17 are all of the same flavor: if $f \in \mathcal{C}_{1}-\mathcal{C}_{2}$ and $f\{A\} \leq_{\mathcal{C}_{2}} A$, witnessed by $\left\langle h_{i}\right\rangle_{i \in \omega}$, where $h_{i}$ has some property infinitely often, then $A$ is not Kurtz random. In each lemma, the arity of $f$ will be $k$ and the arity of $h_{i}$ will be $a_{i}$ for each $i \in \omega$.

Lemma 13. Suppose $\mathcal{C}_{1}$ and $\mathcal{C}_{2}$ are distinct polynomial clones with $f \in \mathcal{C}_{1}-\mathcal{C}_{2}$. For any $A \in \mathbf{n}^{\omega}$, if $f\{A\} \leq_{\mathcal{C}_{2}} A$, witnessed by $\left\langle h_{i}\right\rangle_{i \in \omega} \subseteq \mathcal{C}_{2}$, and $k i+1 \geq a_{i}$ for infinitely many $i$, then $A$ is not Kurtz random.

Proof. Let $n_{0}<n_{1}<\cdots$ be the sequence of all natural numbers such that $a_{n_{i}} \leq k n_{i}+1$ for each $i$. The hypothesis that the sequence $\left\langle h_{i}\right\rangle_{i \in \omega}$ is computable implies that the sequence $\left\langle n_{i}\right\rangle_{i \in \omega}$ is computable. Let $m_{i}=k$ for each $i \in \omega$. According to Lemma 12, it suffices to show that there is a computable function $\Phi: \mathbf{n}^{\omega} \rightarrow \mathbf{n}^{\omega}$ such that

- if $|\sigma|=k n_{i}+1$, then $|\Phi(\sigma)|=k$,

- and if $\sigma=A \uparrow k n_{i}+1$ then $\Phi(\sigma) \neq A\left(k n_{i}+1\right) \cdots A\left(k n_{i}+k\right)$.

Fix $i$ and $\sigma \in \mathbf{n}^{<\omega}$ with $|\sigma|=k n_{i}+1$. Since $f \in \mathcal{C}_{1}-\mathcal{C}_{2}$, it is necessarily nonconstant and whence there is an effectively obtainable length- $k$ string $\tau$ such that $h\left(\sigma\left\lceil a_{i}\right) \neq f(\tau)\right.$. Thus we define $\Phi(\sigma)=\tau$ so that if $\sigma=A(0) \cdots A\left(k n_{i}\right)$, then the assumed equality $h\left(A \uparrow a_{i}\right)=f\left(A\left(k n_{i}+1\right) \cdots A\left(k n_{i}+k\right)\right.$ gives $A\left(k n_{i}\right) \cdots A\left(k n_{i}+k\right) \neq \tau$. If $|\rho| \neq k n_{i}+1$ for each $i$, then we define $\Phi(\rho)=0$. The function $\Phi$ then satisfies the requirements and so the proof is complete.

We state the result obtained in the last paragraph as a corollary for future use.

Corollary 14. Suppose $\mathcal{C}_{1}$ and $\mathcal{C}_{2}$ are distinct polynomial clones with $f \in$ $\mathcal{C}_{1}-\mathcal{C}_{2}$. For any $A \in \mathbf{n}^{\omega}$, if $f\{A\} \leq_{\mathcal{C}_{2}} A$, witnessed by $\left\langle h_{i}\right\rangle_{i \in \omega} \subseteq \mathcal{C}_{2}$, and $n_{0}<$ $n_{1}<\cdots$ is a computable sequence of natural numbers such that $k n_{i}+1 \geq a_{n_{i}}$ for each $i$, then there is a computable function $\Phi: \mathbf{n}^{\omega} \rightarrow \mathbf{n}^{\omega}$ such that

- if $|\sigma|=k n_{i}+1$, then $|\Phi(\sigma)|=k$,

- if $\sigma=A \uparrow k n_{i}+1$ then $\Phi(\sigma) \neq A\left(k n_{i}+1\right) \cdots A\left(k n_{i}+k\right)$.

In addition to $k i+1 \geq a_{i}$ for infinitely many $i$, there are two more cases to consider: $k i+k<a_{i}$ for infinitely many $i$ and $k i+j_{i}=a_{i}$ for infinitely many $i$, where $1<j_{i} \leq k$. We consider the latter case first, in Lemma 15 below. To do so, we employ Lemma 7 to obtain a suitable $f \in \mathcal{C}_{1}-\mathcal{C}_{2}$. 
Lemma 15. Suppose $\mathcal{C}_{1}$ and $\mathcal{C}_{2}$ are distinct polynomial clones with $f \in \mathcal{C}_{1}-\mathcal{C}_{2}$ as in Lemma \%. For any $A \in \mathbf{n}^{\omega}$, if $f\{A\} \leq_{\mathcal{C}_{2}} A$, witnessed by $\left\langle h_{n}\right\rangle$ and $k i+j_{i}=a_{i}$ for infinitely many $i$, where $1<j_{i} \leq k$, then $A$ is not Kurtz random.

Proof. Let $n_{0}<n_{1}<\cdots$ be the sequence of all natural numbers such that $k n_{i}+j_{i}=a_{n_{i}}$ for each $i$, where $1<j_{i} \leq k$. The hypothesis that the sequence $\left\langle h_{i}\right\rangle_{i \in \omega}$ is computable implies that the sequence $\left\langle n_{i}\right\rangle_{i \in \omega}$ is computable. Let $m_{i}=k$ for each $i \in \omega$. According to Lemma 12, it suffices to show that there is a computable function $\Phi: \mathbf{n}^{\omega} \rightarrow \mathbf{n}^{\omega}$ such that

- if $|\sigma|=k n_{i}+1$, then $|\Phi(\sigma)|=k$,

- and if $\sigma=A\left\lceil k n_{i}+1\right.$ then $\Phi(\sigma) \neq A\left(k n_{i}+1\right) \cdots A\left(k n_{i}+k\right)$.

Fix $i$ and $\sigma$ with $|\sigma|=k n_{i}+1$. Using the hypothesis that $f$ is as in Lemma 7, there are strings $x_{1} \cdots x_{k}$ and $y_{j_{i}} \cdots y_{k}$ such that

$$
f\left(x_{1}, \ldots, x_{k}\right) \neq f\left(x_{1}, \ldots, x_{j_{i}-1}, y_{j_{i}}, \ldots, y_{k}\right) .
$$

Thus it cannot be the case that both

$$
\begin{aligned}
& h_{n_{i}}\left(\sigma, x_{1}, \ldots, x_{j_{i}-1}\right)=f\left(x_{1}, \ldots, x_{k}\right) \quad \text { and } \\
& h_{n_{i}}\left(\sigma, x_{1}, \ldots, x_{j_{i}-1}\right)=f\left(x_{1}, \ldots, x_{j_{i}-1}, y_{j_{i}}, \ldots, y_{k}\right),
\end{aligned}
$$

so we assume that $h_{n_{i}}\left(\sigma, x_{1}, \ldots, x_{j_{i}-1}\right) \neq f\left(x_{1}, \ldots, x_{k}\right)$. Let $\tau=x_{1} \cdots x_{k}$. Define $\Phi(\sigma)=\tau$ so that if $\sigma=A(0) \cdots A\left(k n_{i}\right)$, then the assumed equality $h\left(A\left\lceil a_{i}\right)=f\left(A\left(k n_{i}+1\right) \cdots A\left(k n_{i}+k\right)\right.\right.$ gives $A\left(k n_{i}\right) \cdots A\left(k n_{i}+k\right) \neq \tau$. If $|\rho| \neq k n_{i}+1$ for each $i$, then we define $\Phi(\rho)=0$. The function $\Phi$ then satisfies the requirements and so the proof is complete.

We state the result obtained in the last paragraph as a corollary for future use.

Corollary 16. Suppose $\mathcal{C}_{1}$ and $\mathcal{C}_{2}$ are distinct polynomial clones with $f \in$ $\mathcal{C}_{1}-\mathcal{C}_{2}$. For any $A \in \mathbf{n}^{\omega}$, if $f\{A\} \leq_{\mathcal{C}_{2}} A$, witnessed by $\left\langle h_{i}\right\rangle_{i \in \omega} \subseteq \mathcal{C}_{2}$, and $n_{0}<$ $n_{1}<\cdots$ is a computable sequence of natural numbers such that $k n_{i}+j_{i}=a_{n_{i}}$ for some $j_{i}$ for each $i$, then there is a computable function $\Phi: \mathbf{n}^{\omega} \rightarrow \mathbf{n}^{\omega}$ such that

- if $|\sigma|=k n_{i}+1$, then $|\Phi(\sigma)|=k$,

- if $\sigma=A\left\lceil k n_{i}+1\right.$ then $\Phi(\sigma) \neq A\left(k n_{i}+1\right) \cdots A\left(k n_{i}+k\right)$.

The final case to consider is when $k i+k<a_{i}$ for infinitely many $i$.

Lemma 17. Suppose $\mathcal{C}_{1}$ and $\mathcal{C}_{2}$ are distinct polynomial clones with $f \in \mathcal{C}_{1}-\mathcal{C}_{2}$. For any $A \in \mathbf{n}^{\omega}$, if $f\{A\} \leq_{\mathcal{C}_{\in}} A$, witnessed by $\left\langle h_{i}\right\rangle_{i \in \omega} \subseteq \mathcal{C}_{2}$, and $k i+k<a_{i}$ for infinitely many $i$, then $A$ is not Kurtz random. 
Proof. Let $n_{0}<n_{1}<\cdots$ be the sequence of all natural numbers such that $k n_{i}+k<a_{n_{i}}$ for each $i$. The hypothesis that the sequence $\left\langle h_{i}\right\rangle_{i \in \omega}$ is computable implies that the sequence $\left\langle n_{i}\right\rangle_{i \in \omega}$ is computable. Because $\left\langle n_{i}\right\rangle_{i \in \omega}$ is a strictly increasing sequence, we can assume that $a_{n_{i}}-1 \leq k n_{i+1}$ for each $i$. Let $m_{i}=$ $a_{n_{i}}-1-k n_{i}$ for each $i \in \omega$. According to Lemma 12, it suffices to show that there is a computable function $\Phi: \mathbf{n}^{\omega} \rightarrow \mathbf{n}^{\omega}$ such that

- if $|\sigma|=k n_{i}+1$, then $|\Phi(\sigma)|=n^{m_{i}-k} m_{i}$,

- and if $\sigma=A\left\lceil k n_{i}+1\right.$ then $\Phi(\sigma) \uparrow_{(j-1) m_{i}+1}^{j m_{i}} \neq A\left(k n_{i}+1\right) \cdots A\left(k n_{i}+m_{i}\right)$ for $j \in\left\{1, \ldots, n^{m_{i}-k}\right\}$.

Fix $i$ and fix $\sigma \in \mathbf{n}^{<\omega}$ with $|\sigma|=k n_{i}+1$. For any string $\rho$ with $|\rho|=m_{i}-k$, it cannot be the case that $f\left(x_{1}, \ldots, x_{k}\right)=h_{n_{i}}\left(\sigma, x_{1}, \ldots, x_{k}, \rho\right)$ for each string $x_{1} \cdots x_{k}$, for otherwise $f$ is a composition of $h_{n_{i}}$ with constants, contrary to $f \notin \mathcal{C}_{2}$. Thus for each such $\rho$ there is a length- $k$ string $\tau_{\rho}$ such that $f\left(\tau_{\rho}\right) \neq$ $h_{n_{i}}\left(\sigma \tau_{\rho} \rho\right)$. This gives $\alpha:=n^{m_{i}-k}$ strings $\xi_{1}, \ldots, \xi_{\alpha}$ (one for each $\rho$ ) of length $m_{i}$ such that $f\left(\xi_{j}\lceil k) \neq h_{i}\left(\sigma \xi_{j}\right)\right.$ for $j \in\{1, \ldots, \alpha\}$.

Define then $\Phi(\sigma)=\xi_{1} \xi_{2} \cdots \xi_{\alpha}$. Clearly $|\Phi(\sigma)|=n^{m_{i}-k} m_{i}$. Suppose $\sigma=$ $A\left\lceil k n_{i}+1\right.$. Then because $\Phi(\sigma) \uparrow_{\left(j-1 m_{i}+1\right.}^{j m_{i}}=\xi_{j}$, we see that $\xi_{j} \neq A\left(k n_{i}+\right.$ 1) $\cdots A\left(a_{i}-1\right)$ because $h_{i}\left(\sigma \xi_{j}\right) \neq f\left(\xi_{j} \mid k\right)$ but by hypothesis we must have $h_{i}\left(\sigma A\left(k n_{i}+1\right) \cdots A\left(a_{i}-1\right)\right)=f\left(A\left(k n_{i}+1\right) \cdots A\left(k n_{i}+k\right)\right)$.

Defining $\Phi(\sigma)=0$ when $|\sigma| \neq k n_{i}+1$ for any $i$ completes the construction of $\Phi$ and also the proof.

Now we are in position to state the main result of this section.

Theorem 18. Suppose $\mathcal{C}_{1}$ and $\mathcal{C}_{2}$ are distinct polynomial clones on $\mathbf{n}$ with $\mathcal{C}_{1} \nsubseteq \mathcal{C}_{2}$. If $A \in \mathbf{n}^{\omega}$ is Kurtz random, then there is a $B \in \mathbf{n}^{\omega}$ such that $B \leq \mathcal{C}_{1} A$, but $B \not \mathcal{C}_{2} A$.

Proof. Suppose there are polynomial clones $\mathcal{C}_{1} \nsubseteq \mathcal{C}_{2}$ such that $B \leq_{\mathcal{C}_{1}} A \Rightarrow$ $B \leq \mathcal{C}_{2} A$ for each $A, B \in \mathbf{n}^{\omega}$. Then in particular, for $f \in \mathcal{C}_{1}-\mathcal{C}_{2}$ as in Lemma 7 we have $f\{A\} \leq_{\mathcal{C}_{2}} A$. At least one of the cases in Lemmas 13, 15, and 17 must occur infinitely often. But in each case, we shown that $A$ cannot be Kurtz random.

Corollary 19. If $\mathcal{C}_{1} \nsubseteq \mathcal{C}_{2}$, then the set $\mathrm{Eq}\left(\mathcal{C}_{1}, \mathcal{C}_{2}\right)=\left\{A \in \mathbf{n}^{\omega}: \forall B \in \mathbf{n}^{\omega}\left(B \leq_{\mathcal{C}_{1}}\right.\right.$ $\left.\left.A \Rightarrow B \leq_{\mathcal{C}_{2}} A\right)\right\}$ has measure zero.

Proof. Theorem 18 implies that $A \notin \operatorname{Eq}\left(\mathcal{C}_{1}, \mathcal{C}_{2}\right)$ for any Kurtz random $A \in \mathbf{n}^{\omega}$. The corollary to Lemma 10 then implies that $\mu\left(\operatorname{Eq}\left(\mathcal{C}_{1}, \mathcal{C}_{2}\right)\right)=0$.

Beside the set $\operatorname{Eq}\left(\mathcal{C}_{1}, \mathcal{C}_{2}\right)$ being measure-theoretically small (null), it is also topologically small (meager). To prove this we need the following result.

Lemma 20. The collection of all Kurtz random sequences is a countable intersection of a collection of open, measure-one subsets of $\mathbf{n}^{\omega}$. 
Proof. The collection $\mathcal{D}$ of all computable, unbounded, nondecreasing function from $\omega$ into $\omega$ is countable, as is the collection $\mathcal{M}$ of all computable martingales. So for each $d \in \mathcal{D}$ and each $M \in \mathcal{M}$, let $O_{M, d}=\bigcup_{n}\left\{A \in n^{\omega}: M\lceil n<d(n)\}\right.$. For a fixed $M, d$, and $n$ the set $\left\{A \in n^{\omega}: M \uparrow n<d(n)\right\}$ is clopen and so $O_{M, d}$ is open. Finally, $\bigcap_{M, d} O_{M, d}=\left\{A \in n^{\omega}: A\right.$ is Kurtz random $\}$.

Thus the complement of all Kurtz random sequences is a countable union of closed null sets. But any closed null set must be meager because its complement must be dense. Since a subset of meager set is meager, we have the following corollary.

Corollary 21. The set $\operatorname{Eq}\left(\mathcal{C}_{1}, \mathcal{C}_{2}\right)$ is meager.

\section{KL-immunity and KL-stochasticity}

Now we turn our attention to another measure-one subset of $\mathbf{n}^{\omega}$ that is incomparable with the set of Kurtz random sequences but is still contained in $\operatorname{Eq}\left(\mathcal{C}_{1}, \mathcal{C}_{2}\right)^{\complement}$, the Kolmogorov-Loveland (KL) immune sequences and the KL-stochastic sequences. KL-stochasticity (and stochasticity in general) was another attempt to define randomness and has been studied extensively. Our definitions and notations related to it follow Merkle [8] except that we are working over $\mathbf{n}$ instead of $\{0,1\}$. KL-immunity is a concept defined here for the first time that is strictly weaker than KL-stochasticity.

As a warm-up for Definition 22 below, suppose we are playing an infinite game in which an unknown sequence $A \in \mathbf{n}^{\omega}$ is fixed. Our goal is to select bits from $A$ so as to create a new sequence $B \in \mathbf{n}^{\omega}$ such that for some $j \in \mathbf{n}$, the distribution of $j$ 's in $B$ is not uniform; i.e.

$$
\lim _{i \rightarrow \infty} \frac{|\{l<i: B(l)=j\}|}{i} \neq \frac{1}{n},
$$

or that limit does not exist.

The rules stipulate that we can have revealed to us any bits of $A$ we like, but can only select a bit for entry into our sequence if that bit has not already been revealed. Once a bit has been selected for addition to $B$ it also becomes revealed. One subtle yet important point is that the bits of $A$ need not be selected in increasing order, so if $B\left(i_{1}\right)=A\left(j_{1}\right)$ and $B\left(i_{2}\right)=A\left(j_{2}\right)$, then it could be the case that $i_{1}<i_{2}$ but $j_{1}>j_{2}$.

For example, suppose $A \in 2^{\omega}$ and we have (reliable) inside information telling us that (a) $A\left(10^{i}-1\right)=1$ whenever $A\left(10^{i}\right)=1$ and (b) $A\left(10^{i}\right)=1$ for infinitely many $i$. To win the game on said $A$, we would ask to see the bits $A\left(10^{i}\right)$ (one at a time), and each time we see that $A\left(10^{i}\right)=1$, we would add the bit $A\left(10^{i}-1\right)$ to our $B$. The resultant sequence $B$ would then be everywhere 1 , so we win!

An important factor in the game is whether the strategy we follow is computable (as in the above example) or not. Perhaps we first request $A(10)$ to be revealed, and if it is a 1 we put $B(0)=A(11)$ whereas if $A(10)=0$ we put 
$B(0)=A(8)$. Then having now seen either both $A(10)$ and $A(11)$ or both $A(10)$ and $A(8)$, our strategy effectively tells us which bit to either reveal or select. And so forth. Or perhaps we just select bits of $A$ at random and add them to $B$. No computable strategy can select bits at random. No sequence can be impervious to all selection strategies, but nearly all sequences are impervious to all computable selection strategies; such are the Kolmogorov-Loveland stochastic sequences.

Definition 22. A selection rule is a function

$$
\begin{aligned}
s: \mathbf{n}^{<\omega} & \rightarrow \omega \times\{0,1\} \\
\sigma & \mapsto(x(\sigma), b(\sigma))
\end{aligned}
$$

such that if $s(\sigma)$ is defined and $\tau \prec \sigma$, then $s(\tau)$ is defined and $x(\sigma) \neq x(\tau)$.

Given a selection rule $s$ and a sequence $A \in \mathbf{n}^{\omega}$, the the sequence of scanned places $\left\langle x_{i}\right\rangle_{i \in \omega}$ is defined recursively by

$$
\begin{aligned}
x_{0} & =x(\varnothing) \\
x_{i} & =x\left(A\left(x_{0}\right) A\left(x_{1}\right) \cdots A\left(x_{i-1}\right)\right) .
\end{aligned}
$$

The sequence of selected places $\left\langle z_{i}\right\rangle_{i \in \omega}$ is the subsequence of $\left\langle x_{i}\right\rangle_{i \in \omega}$ formed by taking only those $x_{i}$ such that $b\left(A\left(x_{0}\right) A\left(x_{1}\right) \cdots A\left(x_{i-1}\right)\right)=1$.

The subsequence of $A$ that is selected by $s$ is the sequence $A\left(z_{0}\right) A\left(z_{1}\right) \cdots$.

The sequence $A$ is Kolmogorov-Loveland (KL) stochastic if for each partial computable selection rule $s$, the sequence of selected places is finite or the frequency of any element of $\mathbf{n}$ in the subsequence of $A$ selected by $s$ is $1 / n$; i.e.

$$
\lim _{i \rightarrow \infty} \frac{\left|\left\{l<i: A\left(z_{l}\right)=j\right\}\right|}{i}=\frac{1}{n} \quad \text { for each } j \in \mathbf{n} .
$$

Finally $A$ is Kolmogorov-Loveland (KL) immune if for each partial computable selection rule $s$, the sequence of selected places is finite or each element of $\mathbf{n}$ shows up infinitely often in the sequence of selected places; i.e. for each $j \in \mathbf{n}$ the set $\left\{i: A\left(z_{i}\right)=j\right\}$ is infinite.

Clearly KL-stochasticity implies KL-immunity. So when we show below that any sequence in $\operatorname{Eq}\left(\mathcal{C}_{1}, \mathcal{C}_{2}\right)$ is not KL-immune, we are also showing that it is not KL-stochastic.

It is known that there are KL-stochastic sequences that are not Kurtz random as well as Kurtz random sequences that are not stochastic [16]. In fact, there are KL-stochastic sequences with each initial segment having more 1's than 0's [8], a fact that shows why KL-stochasticity does not capture what is intuitively considered "random".

The following simple proposition motivates the use of the word "immune" in KL-immune.

Proposition 23. A sequence $A$ is not KL-immune if and only if there is a partial computable selection rule s such that for some $j \in \mathbf{n}$, the subsequence of A selected by $s$ is infinite and contains no $j$ 's. 
Proof. Suppose $A$ is not KL-immune. Then there is a partial computable selection rules $s$ such that for some $j \in \mathbf{n}$, the subsequence of $A$ selected by $s$ contains only finitely many $j$ 's; i.e. the set $\left\{i: A\left(z_{i}\right)=j\right\}$ is finite. Let $x_{i_{1}}, \ldots, x_{i_{m}}$ be the elements of the sequence of selected places such that $b\left(x_{0}, \ldots, x_{i_{l}-1}\right)=1$ and $A\left(x_{i_{l}}\right)=j$ for each $l \in\{1, \ldots, m\}$. Define $b^{\prime}$ to be identical to $b$ except $b^{\prime}\left(x_{0}, \ldots, x_{i_{l}}\right)=0$ for each $l$. Then $b^{\prime}$ retains $b^{\prime}$ 's computability and the subsequence selected by $s^{\prime}:=\left(x, b^{\prime}\right)$ is then the same as that of $s$, but without j's.

The other direction is trivial.

To show that $A \notin \operatorname{Eq}\left(\mathcal{C}_{1}, \mathcal{C}_{2}\right)$ for any KL-immune sequences $A$, we need the following lemma, which is a KL-immunity analog to Lemma 12.

Lemma 24. Let $A \in \mathbf{n}^{\omega}$ and $k \in \omega^{+}$. Let $n_{0}<n_{1}<\cdots$ be a computable sequence of natural numbers and $F_{0} \subsetneq F_{1} \subsetneq \cdots$ a computable sequence of finite sets of natural numbers with $F_{i}=\left\{\beta_{0}^{i}<\beta_{1}^{i} \cdots<\beta_{z(i)}^{i}\right\}$, (so $\left.z(i)+1=\left|F_{i}\right|\right)$. Suppose that for each $i \in \omega$ the following properties hold:

- $\left\{k n_{i}+1, \ldots, k n_{i}+k\right\} \cap F_{i}=\emptyset$,

- $\left\{k n_{i}+1, \ldots, k n_{i}+k\right\} \subseteq F_{i+1}$,

- $\max F_{i} \leq k n_{i+1}$.

If there is a computable function $\Phi: \mathbf{n}^{\omega} \rightarrow \mathbf{n}^{\omega}$ such that

- if $|\sigma|=\left|F_{i}\right|$ for some $i$, then $|\Phi(\sigma)|=k$,

- if $\sigma=A\left(\beta_{0}^{i}\right) A\left(\beta_{1}^{i} \cdots A\left(\beta_{z(i)}^{i}\right)\right.$ then $\Phi(\sigma) \neq A\left(k n_{i}+1\right) \cdots A\left(k n_{i}+k\right)$,

then $A$ is not $K L$-immune.

To prove Lemma 24, we first informally describe a selection strategy that follows the rules of the above-described game and yields a subsequence $B$ that is nearly void of some $j \in \mathbf{n}$. Then we formally construct the strategy's corresponding selection rule.

Selection strategy. By the pigeon hole principal, there is a string $\sigma=y_{1} \cdots y_{k}$ such that $\Phi\left(A\left(\beta_{0}^{i}\right) A\left(\beta_{1}^{i}\right) \cdots A\left(\beta_{z(i)}^{i}\right)\right)=\sigma$ for infinitely many $i$ and some (possibly empty) prefix $\tau \prec \sigma$, with $\tau y_{j} \cdots y_{k}=\sigma$ such that out of all the $i$ 's where $\Phi\left(A\left(\beta_{0}^{i}\right) A\left(\beta_{1}^{i}\right) \cdots A\left(\beta_{z(i)}^{i}\right)\right)=\sigma$, it is the case that $\tau \prec A\left(k n_{i}+1\right) \cdots A\left(k n_{i}+k\right)$ infinitely often, but $\tau y_{j} \prec A\left(k n_{i}+1\right) \cdots A\left(k n_{i}+k\right)$ only finitely often. Fix such $\sigma$ and $\tau$.

Proceeding with our strategy, for each $i$ we have revealed to us each bit $A(\beta)$ for each $\beta \in F_{i}$ as well as the first $|\tau|$ bits of $A\left(k n_{i}+1\right) \cdots A\left(k n_{i}+k\right)$. If $\Phi\left(A\left(\beta_{0}^{i}\right) A\left(\beta_{1}^{i}\right) \cdots A\left(\beta_{z(i)}^{i}\right)\right)=\sigma$ and we've seen $\tau=A\left(k n_{i}+1\right) \cdots A\left(k n_{i}+j-1\right)$ (where $A\left(k n_{i}+1\right) \cdots A\left(k n_{i}+0\right):=\varnothing$ in case $j=1$ ), then we select $A\left(k n_{i}+j\right)$ for entry into our subsequence and also have the bits $A\left(k n_{i}+j+1\right) \cdots A\left(k n_{i}+k\right)$ revealed. Otherwise we still have $A\left(k n_{i}+j\right) \cdots A\left(k n_{i}+k\right)$ revealed without 
selecting any bits for entry into our subsequence. The subsequence selected then will be infinite, since $\Phi\left(A\left(\beta_{0}^{i}\right) A\left(\beta_{1}^{i}\right) \cdots A\left(\beta_{z(i)}^{i}\right)\right)=\sigma$ for infinitely many $i$. However, by the definition of $\tau$, the subsequence will only have finitely many $y_{j}$ 's.

Remark. The selection strategy above exhibits a common occurrence in computability theory: the algorithm was not given explicitly, but just shown to exist. We had no effective way of determining which $\sigma$ would be ruled out (by $\Phi)$ infinitely often, but knew that some $\sigma$ would be. So we actually constructed a (finite) family of betting strategies, one for each $\sigma \in \mathbf{n}^{k}$.

Selection rule construction. We construct a computable section rule $s: \mathbf{n}^{<\omega} \rightarrow$ $\omega \times\{0,1\}$ corresponding to the strategy just outlined.

Let $\sigma$ and $\tau$ be as in the selection strategy, so that $\tau=\sigma\lceil j-1$ and among the infinitely many $i$ such that such that $\Phi\left(A\left(\beta_{0}^{i}\right) A\left(\beta_{1}^{i}\right) \cdots A\left(\beta_{z(i)}^{i}\right)\right)=\sigma$ it is the case that $\tau \prec A\left(k n_{i}+1\right) \cdots A\left(k n_{i}+k\right)$ infinitely often, but $\tau y_{j} \prec$ $A\left(k n_{i}+1\right) \cdots A\left(k n_{i}+k\right)$ only finitely often.

Let $m(0)=z(0)$ and $\alpha_{s}^{0}=\beta_{s}^{0}$ for $s \in\{0,1, \ldots, z(0)\}$. For each $i>0$ let

$$
F_{i}-\bigcup_{s<i}\left(F_{s} \cup\left\{k n_{s}+1, k n_{s}+2, \ldots, k n_{s}+k\right\}\right)=\left\{\alpha_{0}^{i}<\alpha_{1}^{i} \cdots \alpha_{m(i)}^{i}\right\} .
$$

The hypothesis that $F_{i}$ is computable implies that $\alpha_{t}^{i}$ and $\beta_{t}^{i}$ are computable functions of $i$ and $t$ and $m(i)$ and $z(i)$ are computable functions of $i$.

Since a selection rule $s$ is determined by its components, $s(\rho)=(x(\rho), b(\rho))$ for each string $\rho$, we construct the functions $x: \mathbf{n}^{<\omega} \rightarrow \omega$ and $b: \mathbf{n}^{<\omega} \rightarrow\{0,1\}$ simultaneously. The construction is carried out recursively on $i$, as in $n_{i}$.

First, for $|\rho| \leq m(0)$ define

$$
x(\rho)=\alpha_{|\rho|}^{0} \quad \text { and } \quad b(\rho)=0 .
$$

Fix $\rho$ with $|\rho|=m(0)+1$ and let $\Phi(\rho)=\xi$. If $A\left(\alpha_{0}^{0}\right) A\left(\alpha_{1}^{0}\right) \cdots A\left(\alpha_{m(0)}^{0}\right)=\rho$, then by hypothesis $A\left(k n_{0}+1\right) \cdots A\left(k n_{0}+k\right) \neq \xi$. For each string $v$ with $0 \leq|v|<k$ define

$$
x(\rho v)=k n_{0}+|v|+1 \quad \text { and } \quad b(\rho v)= \begin{cases}0 & \text { if } v \neq \tau \text { or } \xi \neq \sigma \\ 1 & \text { if } v=\tau \text { and } \xi=\sigma\end{cases}
$$

and for each $v$ with $|v|=k$, define

$$
x(\rho v)=\alpha_{0}^{1} \quad \text { and } \quad b(\rho v)=0 .
$$

At this point $x$ and $b$ are defined for all words of length at most $m(0)+1+k=$ $z(1)-m(1)$ and $x(\xi)=\alpha_{0}^{1}$ whenever $|\xi|=z(1)-m(1)$.

Suppose now that $x$ and $b$ have been defined for all words of length at most $p:=z(i)-m(i)$ and $x(\rho)=\alpha_{0}^{i}$ whenever $|\rho|=p$. For $|\rho|=p$ and $|v| \leq m(i)$ define

$$
x(\rho v)=\alpha_{|v|}^{i} \quad \text { and } \quad b(\rho v)=0 .
$$


Fix $v$ with $|v|=m(i)+1$. Let $\Phi(\rho v)=\xi$. If $A\left(\beta_{0}^{i}\right) A\left(\beta_{1}^{i}\right) \cdots A\left(\beta_{z(i)}^{0}\right)=\rho v$, then by hypothesis $A\left(k n_{i}+1\right) \cdots A\left(k n_{i}+k\right) \neq \xi$. For each string $\nu$ with $0 \leq|\nu|<k$ define

$$
x(\rho v \nu)=k n_{i}+|\nu|+1 \quad \text { and } \quad b(\rho v \nu)= \begin{cases}0 & \text { if } \nu \neq \tau \text { or } \xi \neq \sigma \\ 1 & \text { if } \nu=\tau \text { and } \xi=\sigma,\end{cases}
$$

and for each $\nu$ with $|\nu|=|\sigma|$, define

$$
x(\rho v \nu)=\alpha_{0}^{1} \quad \text { and } \quad b(\rho v \nu)=0 .
$$

So $x$ and $b$ are now defined for all words of length at most $z(i)-m(i)+m(i)+$ $1+|\sigma|=z(i)+1+|\sigma|=z(i+1)-m(i+1)$. This completes the recursive construction of $x$ and $b$, and hence of $s$ as well. Our next and final aim is to show that the subsequence of $A$ that is selected by $s$ is infinite yet contains only finitely many $y_{j}$ 's.

Since the definition of $x(\rho)$ depends only on the length of $\rho$, the sequence of scanned places $\left\langle x_{i}\right\rangle_{i \in \omega}$ is given by

$$
\alpha_{0}^{0}, \ldots, \alpha_{m(0)}^{0}, k n_{0}+1, \ldots, k n_{0}+k, \alpha_{0}^{1}, \ldots, \alpha_{m(1)}^{1}, k n_{1}+1, \ldots, k n_{1}+k, \alpha_{0}^{2}, \ldots
$$

The construction of $b$ then gives that $b\left(A\left(x_{0}\right) \cdots A\left(x_{i}\right)\right)=1$ if and only if $A\left(x_{0}\right) \cdots A\left(x_{i}\right)=v A\left(\alpha_{m(s)}^{s}\right) \tau$ for some $s$ and some string $v$. By hypothesis, that will happen for infinitely many $i$, and for only finitely many of those $i$ will $A\left(x_{i+1}\right)=y_{j}$, since since $x_{i+1}=k n_{s}+|\tau|+1$.

Lemma 25. Suppose $\mathcal{C}_{1}$ and $\mathcal{C}_{2}$ are distinct polynomial clones with $f \in \mathcal{C}_{1}-\mathcal{C}_{2}$. For any $A \in \mathbf{n}^{\omega}$, if $f\{A\} \leq_{\mathcal{C}_{2}} A$, witnessed by $\left\langle h_{i}\right\rangle_{i \in \omega} \subseteq \mathcal{C}_{2}$, and $k i+1 \geq a_{i}$ for infinitely many $i$, then $A$ is not KL-immune.

Proof. Let $n_{0}<n_{1} \cdots$ be the places where $k n_{i}+1 \geq a_{n_{i}}$. The sequence $\left\langle n_{i}\right\rangle_{i \in \omega}$ is computable because $\left\langle h_{i}\right\rangle_{i \in \omega}$ is. Let $F_{i}=\left\{0,1,2, \ldots, k n_{i}\right\}$. Then $F_{0} \subsetneq F_{1} \subsetneq \cdots$ and the sequence $\left\langle F_{i}\right\rangle_{i \in \omega}$ is computable because $\left\langle n_{i}\right\rangle_{i \in \omega}$ is. Clearly the properties

- $\left\{k n_{i}+1, \ldots, k n_{i}+k\right\} \cap F_{i}=\emptyset$,

- $\left\{k n_{i}+1, \ldots, k n_{i}+k\right\} \subseteq F_{i+1}$, and

- $\max F_{i} \leq k n_{i+1}$

hold for each $i \in \omega$. Thus by Lemma 24, it suffices to show that there is a computable function $\Phi: \mathbf{n}^{\omega} \rightarrow \mathbf{n}^{\omega}$ such that

- if $|\sigma|=\left|F_{i}\right|=k n_{i}+1$ for some $i$, then $|\Phi(\sigma)|=k$,

- if $\sigma=A\left\lceil k n_{i}+1\right.$, then $\Phi(\sigma) \neq A\left(k n_{i}+1\right) \cdots A\left(k n_{i}+k\right)$.

This is exactly the result of Corollary 14 . 
Lemma 26. Suppose $\mathcal{C}_{1}$ and $\mathcal{C}_{2}$ are distinct polynomial clones with $f \in \mathcal{C}_{1}-\mathcal{C}_{2}$ as in Lemma 7. For any $A \in \mathbf{n}^{\omega}$, if $f\{A\} \leq_{\mathcal{C}_{2}} A$, witnessed by $\left\langle h_{n}\right\rangle$ and $k i+j_{i}=a_{i}$ for infinitely many $i$, where $1<j_{i} \leq k$, then $A$ is not $K L$-immune.

Proof. Let $n_{0}<n_{1} \cdots$ be the places where $k n_{i}+j_{i}=a_{n_{i}}$ for some $1<$ $j_{i} \leq k$. The sequence $\left\langle n_{i}\right\rangle_{i \in \omega}$ is computable because $\left\langle h_{i}\right\rangle_{i \in \omega}$ is. Let $F_{i}=$ $\left\{0,1,2, \ldots, k n_{i}\right\}$. Then the sequence $\left\langle F_{i}\right\rangle_{i \in \omega}$ is computable because $\left\langle n_{i}\right\rangle_{i \in \omega}$ is and clearly $F_{0} \subseteq F_{1} \subseteq \cdots$. Clearly the properties

- $\left\{k n_{i}+1, \ldots, k n_{i}+k\right\} \cap F_{i}=\emptyset$,

- $\left\{k n_{i}+1, \ldots, k n_{i}+k\right\} \subseteq F_{i+1}$, and

- $\max F_{i} \leq k n_{i+1}$

hold for each $i \in \omega$. Thus by Lemma 24, it suffices to show that there is a computable function $\Phi: \mathbf{n}^{\omega} \rightarrow \mathbf{n}^{\omega}$ such that

- if $|\sigma|=\left|F_{i}\right|=k n_{i}+1$ for some $i$, then $|\Phi(\sigma)|=k$,

- if $\sigma=A\left\lceil k n_{i}+1\right.$, then $\Phi(\sigma) \neq A\left(k n_{i}+1\right) \cdots A\left(k n_{i}+k\right)$.

This is exactly the result of Corollary 16 .

Lemma 27. Suppose $\mathcal{C}_{1}$ and $\mathcal{C}_{2}$ are distinct polynomial clones with $f \in \mathcal{C}_{1}-\mathcal{C}_{2}$. For any $A \in \mathbf{n}^{\omega}$, if $f\{A\} \leq_{\mathcal{C}_{2}} A$, witnessed by $\left\langle h_{i}\right\rangle_{i \in \omega} \subseteq \mathcal{C}_{2}$, and $k i+k<a_{i}$ for infinitely many $i$, then $A$ is not KL Stochastic.

Proof. Let $n_{0}<n_{1} \cdots$ be the places where $k n_{i}+k<a_{n_{i}}$. The sequence $\left\langle n_{i}\right\rangle_{i \in \omega}$ is computable because $\left\langle h_{i}\right\rangle_{i \in \omega}$ is. By passing to a subsequence, we can assume that $a_{i} \leq k n_{i+1}+1$ for each $i$. Set $F_{i}=\left\{0,1,2, \ldots, a_{n_{i}}-1\right\}-\left\{k n_{i}+\right.$ $\left.1, \ldots, k n_{i}+k\right\}$. Then the sequence $\left\langle F_{i}\right\rangle_{i \in \omega}$ is computable because $\left\langle n_{i}\right\rangle_{i \in \omega}$ is. The assumption that $a_{i} \leq k n_{i+1}+1$ for each $i$ implies $F_{0} \subseteq F_{1} \subseteq \cdots$. Clearly the properties

- $\left\{k n_{i}+1, \ldots, k n_{i}+k\right\} \cap F_{i}=\emptyset$,

- $\left\{k n_{i}+1, \ldots, k n_{i}+k\right\} \subseteq F_{i+1}$, and

- $\max F_{i} \leq k n_{i+1}$

hold for each $i \in \omega$. Thus by Lemma 24, it suffices to show that there is a computable function $\Phi: \mathbf{n}^{\omega} \rightarrow \mathbf{n}^{\omega}$ such that

- if $|\sigma|=\left|F_{i}\right|=a_{n_{i}}-k$ for some $i$, then $|\Phi(\sigma)|=k$,

- if $\sigma=A(0) A(1) \cdots A\left(k n_{i}\right) A\left(k n_{i}+k+1\right) \cdots A\left(a_{i}-1\right)$ then $\Phi(\sigma) \neq A\left(k n_{i}+\right.$ 1) $\cdots A\left(k n_{i}+k\right)$ 
Constructing this $\Phi$ is a slight modification of the construction of $\Phi$ in Lemma 17.

Fix $i$ and fix strings $\tau=y_{0} y_{1} \cdots y_{k n_{i}}$ and $\rho=y_{k n_{i}+k+1} \cdots y_{a_{i}}$. Let $\sigma=\tau \rho$, so that $|\sigma|=a_{n_{i}}-k$. It cannot be the case that $f\left(x_{1}, \ldots, x_{k}\right)=h_{n_{i}}\left(\tau, x_{1}, \ldots, x_{k}, \rho\right.$ for each string $x_{1} \cdots x_{k}$, for otherwise $f$ is a composition of $h_{n_{i}}$ with constants, contrary to $f \notin \mathcal{C}_{2}$. Thus there is a length- $k$ string $v_{\sigma}$ such that $f\left(v_{\sigma}\right) \neq$ $h_{n_{i}}\left(\tau v_{\sigma} \rho\right)$. Define $\Phi(\sigma)=v_{\sigma}$. It's not hard to see that $\Phi$ satisfies the required properties, so by defining $\Phi(\sigma)=0$ whenever $|\sigma| \neq a_{n_{i}}-k$ for all $i$, we have our $\Phi$.

Via Lemmas 25, 26, and 27 we have exhausted all cases for the relationship between $A(0) \cdots A\left(a_{i}\right)$ and $A(k i+1) \cdots A(k i+k)$. Thus we have Theorem 28 .

Theorem 28. Suppose $\mathcal{C}_{1}$ and $\mathcal{C}_{2}$ are distinct polynomial clones on $\mathbf{n}$ with $\mathcal{C}_{1} \not \subset \mathcal{C}_{2}$. If $A \in \mathbf{n}^{\omega}$ is KL-immune, then there is a $B \in \mathbf{n}^{\omega}$ such that $B \leq \mathcal{C}_{1} A$, but $B \Varangle_{\mathcal{C}_{2}} A$.

And we again get the same result as Corollary 19 .

Corollary 29. If $\mathcal{C}_{1} \nsubseteq \mathcal{C}_{2}$, then the set $\mathrm{Eq}\left(\mathcal{C}_{1}, \mathcal{C}_{2}\right)$ has measure zero.

Proof. Theorem 28 implies that $A \notin \mathrm{Eq}\left(\mathcal{C}_{1}, \mathcal{C}_{2}\right)$ for any KL-immune $A \in \mathbf{n}^{\omega}$. The collection of KL-immune sequences has measure one, since it contains the collection of KL-stochastic sequences, which has measure one [8]. The result follows.

\section{Additional Results}

We have so far been focused on the complement of $\operatorname{Eq}\left(\mathcal{C}_{1}, \mathcal{C}_{2}\right)$, those sequences $A$ such that each distinct polynomial clone yields a different reducibility with respect to $A$; i.e. those $A$ for which $\mathcal{C}_{1} \nsubseteq \mathcal{C}_{2}$ implies the existence of a $B$ such that $B \leq_{\mathcal{C}_{1}} A$ but $B \leq_{\mathcal{C}_{2}} A$. Now we show that are sequences at the other end of the spectrum, sequences $A$ such that all polynomial clones yield the same reducibility with respect to $A$; i.e. there are sequences $A$ such that $\mathcal{C}_{1} \neq \mathcal{C}_{2}$ implies $B \leq_{\mathcal{C}_{1}} A \Leftrightarrow B \leq_{\mathcal{C}_{2}} A$ for each $B$. This is not a new result, but rather an application of a known result (for $n=2$ ) to this generalized situation (where $n$ is arbitrary).

Assume $\left\langle g_{i}\right\rangle_{i \in \omega}$ is an effective enumeration of all operations on $\mathbf{n}$. The polynomial clone consisting of all operations of $\mathbf{n}$ is denoted $\mathcal{C}_{\max }$ (so $\left\langle g_{i}\right\rangle_{i \in \omega}$ is an effective enumeration of $\mathcal{C}_{\text {max }}$ ). The polynomial clone consisting only of the constant and projection functions is denoted $\mathcal{C}_{\text {min }}$.

Definition 30 (Odifreddi [10]). The truth-table cylindrification of $A \in \mathbf{n}^{\omega}$, denoted $A^{t t}$ is the sequence given by

$$
A^{t t}(i)=g_{i}\left(A(0), \ldots, A\left(k_{i}\right)\right)
$$

for each $i$. 
Lemma 31. For any $A \in \mathbf{n}^{\omega}$, the following reduction relationships between $A$ and $A^{\text {tt }}$ hold:

a. $A \leq_{\mathcal{C}_{\min }} A^{t t}$

b. $A^{\text {tt }} \leq_{\mathcal{C}_{\max }} A$.

Proof.

a. For any $i, A(i)=\pi_{i+1}^{i+1}(A(0), \ldots, A(i))$, so if $\pi_{i+1}^{i+1}=g_{j}$ then $A(i)=A^{t t}(j)$ and hence to construct the sequence $\left\langle h_{i}\right\rangle_{i \in \omega}$ that witnesses $A \leq_{\mathcal{C}_{\min }} A^{t t}$, we set $h_{i}=\pi_{j+1}^{j+1}$.

b. Immediate from the definition.

Lemma 32. For any $A \in \mathbf{n}^{\omega}$, if $B \leq_{\mathcal{C}_{\max }} A^{t t}$, then $B \leq_{\mathcal{C}_{\min }} A^{t t}$.

Proof. If $B \leq_{\mathcal{C}_{\max }} A^{t t}$, then by Lemma 31b., $B \leq_{\mathcal{C}_{\max }} A$. Thus there is a computable sequence $\left\langle h_{i}\right\rangle_{i \in \omega}$ that witnesses $B \leq_{\mathcal{C}_{\max }} A$. But if $h_{i}=g_{j_{i}}$, then $B(i)=A^{t t}\left(j_{i}\right)$, so the sequence whose $i^{\text {th }}$ function is $\pi_{j_{i}+1}^{j_{i}+1}$ witnesses $B \leq \mathcal{C}_{\min }$ $A^{t t}$.

Theorem 33. For any $A$ and any distinct polynomial clones $\mathcal{C}_{1}$ and $\mathcal{C}_{2}$, if $B \leq_{\mathcal{C}_{1}} A^{t t}$, then $B \leq_{\mathcal{C}_{2}} A^{t t}$.

Proof. If $B \leq_{\mathcal{C}_{1}} A^{t t}$, then $B \leq_{\mathcal{C}_{\max }} A^{t t}$, so by Lemma $32, B \leq_{\mathcal{C}_{\min }} A^{t t}$ and whence $B \leq \mathcal{C}_{2} A^{t t}$.

For any polynomial clone $\mathcal{C}$, letting $\equiv_{\mathcal{C}}$ be the equivalence relation induced by $\leq_{\mathcal{C}}$ gives a fact about how many sequences (in both the measure-theoretic and topological sense) can be $\mathcal{C}_{\text {min-equivalent to } t} t$ cylinders.

Corollary 34. The set $\left\{B \in \mathbf{n}^{\omega}: \exists A\left(B \equiv_{\mathcal{C}_{\min }} A^{\text {tt }}\right)\right\}$ has measure zero and is meager.

Proof. By the proof of Theorem 33, the given set is contained in $\operatorname{Eq}\left(\mathcal{C}_{1}, \mathcal{C}_{2}\right)$ for any polynomial clones $\mathcal{C}_{1}$ and $\mathcal{C}_{2}$. By Corollary 19 , the set $\operatorname{Eq}\left(\mathcal{C}_{1}, \mathcal{C}_{2}\right)$ has measure zero whenever $\mathcal{C}_{1} \nsubseteq \mathcal{C}_{2}$. By Corollary 21, the set $\operatorname{Eq}\left(\mathcal{C}_{1}, \mathcal{C}_{2}\right)$ is meager whenever $\mathcal{C}_{1} \nsubseteq \mathcal{C}_{2}$. The result then follows from the fact that the collection of null sets and the collection of meager sets are each closed under the subset operation.

\section{Open Questions}

The uses of Kurtz randomness and KL-immunity to show that $\mu\left[\operatorname{Eq}\left(\mathcal{C}_{1}, \mathcal{C}_{2}\right)\right]=0$ whenever $\mathcal{C}_{1} \nsubseteq \mathcal{C}_{2}$ were conspicuously similar. In both cases, we considered the relationship between $A(0) \cdots A\left(a_{i}\right)$ and $A\left(k n_{i}+1\right) \cdots A\left(k n_{i}+k\right)$. So a natural goal is to seek a common refinement of the concepts of KL-immunity and Kurtz randomness. In other words, we would like to find a set $P \subseteq \mathbf{n}^{\omega}$ definable as a 
sort of "randomness" so that all Kurtz random sequences and all KL-immune sequences are in $P$ and for any $A \in P$ and any polynomial clones $\mathcal{C}_{1} \nsubseteq \mathcal{C}_{2}$, there is a $B \in \mathbf{n}^{\omega}$ such that $B \leq_{\mathcal{C}_{1}} A$, but $B \leq_{\mathcal{C}_{2}} A$. We have unsuccessfully tried to find such a $P$ but will not expound here on our fruitless attempts.

Another question we left open regards KL-immunity and -stochasticity. The "KL" refers to the fact that our selection rule need not be monotonic; we can look at any bits of the sequence we want prior to selection. However some definitions of stochasticity require that we look at the bits in order, so that in order to decide if we want to select a bit, we can only reveal (at most) the bits preceding. It is therefore natural to wonder if the "KL" can be removed from our results, so $\operatorname{Eq}\left(\mathcal{C}_{1}, \mathcal{C}_{2}\right)$ is contained in the complement of the collection of all sequences from which a subsequence failing the law of large numbers cannot be selected by a computable monotonic selection rule. Or, more generally, is $\operatorname{Eq}\left(\mathcal{C}_{1}, \mathcal{C}_{2}\right)$ contained in the complement of the collection of all sequences from which a subsequence void of a specific element of $\mathbf{n}$ cannot be selected by a computable monotonic selection rule? Again, our attempts to answer this question has been unsuccessful. It should be noted, however, that because the seven polynomial clones over $\{0,1\}$ are completely known, it might be easy to answer both questions in the case $n=2$ by simply doing a pairwise comparison of the $\left(\begin{array}{l}7 \\ 2\end{array}\right)$ possibilities for $\left(\mathcal{C}_{1}, \mathcal{C}_{2}\right)$.

\section{References}

[1] I. Ágoston, J. Demetrovics, and L. Hannák, On the number of clones containing all constants (a problem of R. McKenzie), Lectures in universal algebra (Szeged, 1983), Colloq. Math. Soc. János Bolyai, vol. 43, NorthHolland, Amsterdam, 1986, pp. 21-25. MR MR860252 (88a:08005)

[2] Valeriy K Bulitko, Reducibility by linear Zhegalkin tables, Siberian Mathematical Journal, Russian (1980), no. 21, 21-332.

[3] Nigel J. Cutland, Computability: An introduction to recursive function theory, Cambridge University Press, 1983.

[4] Alexander Degtev, Comparison of linear reducibility with other reducibilities of tabular type, Algebra and Logic, Russian (1982), no. 21, 21-339.

[5] Rod Downey, Denis R. Hirschfeldt, André Nies, and Sebastiaan A. Terwijn, Calibrating randomness, Bull. Symbolic Logic 12 (2006), no. 3, 411-491. MR MR2248591 (2007j:03055)

[6] Carl G. Jockusch, Jr., Semirecursive sets and positive reducibility, Trans. Amer. Math. Soc. 131 (1968), 420-436.

[7] A. H. Lachlan, Some notions of reducibility and productiveness, Z. Math. Logik Grundlagen Math. 11 (1965), 17-44. MR MR0172795 (30 \#3014) 
[8] Wolfgang Merkle, The Kolmogorov-Loveland stochastic sequences are not closed under selecting subsequences, Journal of Symbolic Logic 68 (2002), $1362-1376$.

[9] André Nies, Computability and randomness, Oxford Logic Guides, xv + 443, Oxford University Press, 2009.

[10] Piergiorgio Odifreddi, Classical recursion theory (volume I), North-Holland Publishing Co., Amsterdam, 1989.

[11] _ Reducibilities, Handbook of Computability Theory (Edward R. Griffor, ed.), Studies in Logic and the Foundations of Mathematics, Elsevier Science B.V., 1999, pp. 98-102.

[12] Emil Post, The two-valued iterative systems of mathematical logic, Annals of Mathematics Studies, no. 5, 1941.

[13] Joseph J. Rotman, An introduction to the theory of groups, Springer-Verlag, New York, 1994.

[14] Halsey Royden, Real analysis, 3rd ed., Prentice Hall, New York, NY, 1988.

[15] Claus Peter Schnorr, Zufälligkeit und wahrscheinlichkeit, Lecture Notes in Mathematics. 1.3, Springer Verlag, 1971.

[16] Yongge Wang, Randomness and complexity, Ph.D. thesis, University of Heidelberg, 1996. 